Article

\title{
On the Emergence of Spacetime and Matter from Model Sets
}

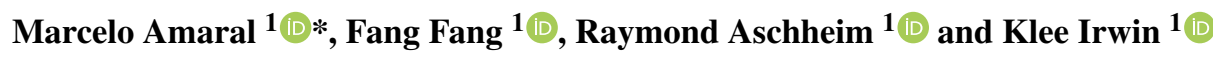 \\ 1 Quantum Gravity Research, Los Angeles, CA 90290, USA \\ * Correspondence: Marcelo@quantumgravityresearch.org
}

\begin{abstract}
Starting from first principles and the mathematics of model sets we propose a framework where emergence of spacetime and matter can be addressed.
\end{abstract}

Keywords: Quantum Gravity; Problem of Time; Particle Physics; Emergence; Holographic Principle; Model Sets

\section{Introduction}

Emergence of spacetime geometry from underlying (Planck scale) discrete structures has being considered recently from different approaches such as entropic gravity [1,2], spin foams [3-5], causal dynamical triangulation [6,7], causal sets [8,9] and multiway hypergraph systems [10-13]. But there is no clear solution to the problem and we know little about the emergence of matter, in terms of particle and fields properties [14], especially in an unified framework. We propose model sets as such a framework.

We consider quasicrystals defined by the geometric cut-and-projection method in its most general form, called model sets [15] (sometimes called cut and projection set), as the underlying mathematical structure under which emergence will be addressed. We agree with [16] on the priority and greater generality of the term "model set" with regard to aperiodic structures and will use it from now on. In quasicrystal mathematical literature, model sets are usually developed as models for quasicrystals materials with good agreement with experiment [15,17-19]. Here we consider the same mathematical structures on much smaller scales. Using the notion of geometric state sums, [20] we define a scale invariant probabilistic measure, which allows us to compute the expected values of observables. Then, the model sets background structure works as a fundamental clock [21,22] for relational evolution of emergent quantum matter.

This paper is organized as follows: in Section 2 we introduce the concept and definitions of model sets. In Section 3 we review and discuss elements of state sums from model sets and the proposed expected values are discussed. We present discussions and implications for emergence of matter and relational quantum evolution in Section 4.

\section{Model Sets Definitions}

We'll start with some mathematical foundations for quasicrystals [15] relevant to our discussion. A cut-and-project scheme (CPS) is a 3 -tuplet $\mathcal{G}=\left(\mathbb{R}^{d}, G, \mathcal{L}\right)$, where $\mathbb{R}^{d}$ is a real euclidean space, $G$ is some locally compact abelian group (in general it can be any topological group) and $\mathcal{L}$ is a lattice

check for

updates

Citation: . Preprints 2021, 1,0 .

https://doi.org/

Received:

Accepted:

Published:

Publisher's Note: MDPI stays neutral with regard to jurisdictional claims in published maps and institutional affiliations. in $\mathbb{R}^{d} \times G$, with the two natural projections $\pi: \mathbb{R}^{d} \times G \rightarrow \mathbb{R}^{d}$ and $\pi_{\perp}: \mathbb{R}^{d} \times G \rightarrow G$. With $L=\pi(\mathcal{L})$, $\pi$ is a bijection between $\mathcal{L}$ and $L$, and $\pi_{\perp}(\mathcal{L})$ dense in $G . \mathcal{E}=\mathbb{R}^{d} \times G$ is the embedding space, the space $\mathbb{R}^{d}$ is called the parallel or physical space and $G$ is the perpendicular or internal space. Due the bijection between $\mathcal{L}$ and $L$, this scheme has a well-defined map, called star map, $\star: L \rightarrow G$ :

$$
x \mapsto x^{\star}:=\pi_{\perp}\left(\pi^{-1}(x)\right) .
$$

The $\star$-image of $L$ is denoted $L^{\star}$. From now on we will restrict the internal space to be another real euclidean space $G=\mathbb{R}^{d}$.

For a given CPS $\mathcal{G}$ and a non-empty relatively compact subset $K \subset G$, the projected set

$$
\triangle(K):=\left\{x \in L \mid x^{\star} \in K\right\}=\left\{\pi(y) \mid y \in \mathcal{L}, \pi_{\perp}(y) \in K\right\},
$$

or any translate $t+\Delta(K)$ with $t \in \mathbb{R}^{d}$, is called model set. $K$ is called the window or the coding set. 
The window $K$ need to be fixed, but it is, in general, arbitrary so that there are an infinity of model sets for a given $\mathcal{G}$. Some properties are representative of model sets $\Delta$, which we review in Appendix A. Within a model set we can have different tiling configurations $\mathcal{T}_{\Delta}$ as reviewed in Appendix A.2. In this case, given a $x_{i} \in \Delta$ and a tiling $\mathcal{T}_{\Delta}$, it can be associated to $x_{i}$ different configurations of prototiles around it, called vertex types $V T_{i}$, with its associated cluster window $K_{V T_{i}}$. A space-filling tiling will be give by a finite set of $V T$ s.

For completeness and to set the notation for the following presentation, we give a general outline in Appendix A for model sets and their associated patterns, tilings and symmetry. Within a model set the cluster $V T_{i}$ at some positions $x_{i}$ can be used as a fixed point, the homothetic (or similarity) center $(\kappa)$, for a local inflation-deflation symmetry (LIDS) transformation Eq. (A10). The general linear transformation preserving distance and solid shapes are the well known orthogonal transformations of $O(n)$, restricted to $S O(n)$ if they preserve orientation. Mixing them with rescaling, or zoom-in zoom-out, diagonal transformations, namely homotheties, we get the similarities. And if we let the scale factor be different in each perpendicular direction, we get the whole $G L(n)$ group of transformation, extended to the affine transformation by mixing with translations. This fixed point transformation that generates mutually locally derivable (MLD) invariant tilings or point sets (see Appendix A.2 for details) can be implemented equivalently by centering the window in $x_{i}^{\star}$ and contracting the window by a new appropriated inflation multiplier $\lambda_{\kappa}$, generating the inflation window $K_{\kappa}=\lambda_{K} K$ (deflation being implemented by a deflation window $K_{K^{d}}=\lambda_{\kappa}^{-1} K$ ). More generally we can consider an inflation window on any $n$-inflation level $K_{\kappa}^{n}=\lambda_{\kappa}^{n} K$. In this case we call this subset of $\Delta$ the $\kappa$-inflation model set defined in any level $n$

$$
\triangle_{\kappa}^{n}:=\Delta\left(K_{K}^{n}\right)=\left\{x \in \Delta \mid x^{\star} \in K_{\kappa}^{n}\right\}=\left\{\pi(y) \mid y \in \mathcal{L}, \pi_{\perp}(y) \in K_{K}^{n}\right\} .
$$

Relative frequency of VTs at level $n$, given from Eq. (A1) in terms of ratios of window polytope volumes, is

$$
f_{\triangle_{\kappa}^{n}}^{r}\left(V T^{n}\right)=\frac{\operatorname{Vol}\left(K_{V T^{n}}\right)}{\operatorname{Vol}\left(K_{\kappa}^{n}\right)} .
$$

This also applies to patterns and results in most cases non-space-filling $\kappa$-inflation tilings (see Appendix A.2, Remark A2). To get the absolute frequency of the $V T$, we take $\operatorname{dens}\left(\Delta_{\kappa}^{n}\right) f_{\Delta_{\kappa}^{n}}^{r}\left(V T^{n}\right)$. Note that the absolute frequency depends on the scale $\left(l_{g}\right)$ of $\mathcal{G}$, but is an MLD invariant, so that it is invariant under LIDS - an invariant in the self-similar space of $\kappa$-inflations.

Given two clusters $\kappa_{i}$ and $\kappa_{j}$ and its $\kappa_{i}$-inflation model sets, at levels $n$ and $m, \Delta_{\kappa_{i}}^{n}$ and $\triangle_{\kappa_{j}}^{m}$, the overlap of their inflation point sets are called hits $\Delta_{H_{i j}}$. Their inflation windows overlap is given by $K_{\kappa_{i j}}=K_{\kappa_{i}}^{n} \cap K_{\kappa_{j}}^{m}$. The hits is then given by

$$
\triangle_{H_{i j}}:=\Delta\left(K_{\kappa_{i j}}\right)=\left\{x \in\left(\Delta_{\kappa_{i}}^{n} \cap \triangle_{\kappa_{j}}^{m}\right)\right\}=\left\{\pi(y) \mid y \in \mathcal{L}, \pi_{\perp}(y) \in K_{\kappa_{i j}}\right\},
$$

A measure of the global overlap at the inflation level $n, m$ is given in terms of their relative frequency, relative to some other level $l$ in the stack

$$
H_{i j}^{K}=f_{\Delta_{K}^{l}}^{a}\left(\triangle_{H_{i j}}\right)=\operatorname{dens}\left(\triangle_{K}^{l}\right) \frac{\operatorname{Vol}\left(K_{K_{i j}}\right)}{\operatorname{Vol}\left(K_{K}^{l}\right)} .
$$

\section{Geometric State Sum Models from Model Sets and Expected Values}

The object of interest here is analogous to the partition function in context of statistic mechanics and thermodynamics, or the path integral in quantum mechanics. But in a discrete setting we move from integration to sums and, without a prior notion of Hamiltonian or Lagrangian (and associated Gibbs measures), we directly specify the weights or amplitudes, where here we focus on weights and can define a measure. With a more general measure for the model set structures we will later discuss limits where one recovers measures with an usual Hamiltonian. To specify the configuration of states of model sets $\Delta$ for a given $\mathcal{G}$ and associated weights we use the constraints given by four principles. We will leave the fourth for the section 3.1 and start with the three principles: 
1- Superposition: Given different tilings (space or non-space-filling) configurations $\mathcal{T}_{\Delta}^{k}$, there are weights $W_{\mathcal{T}_{\Delta}^{k}}$ associated with them and we consider the dynamics states sum

$$
W_{\mathcal{T}_{\Delta}}=\sum_{\mathcal{T}_{\Delta}^{k} \sqsubset \mathcal{T}_{\Delta}} W_{\mathcal{T}_{\Delta}^{k}} .
$$

2- Locality: weight is mainly encoding local information. The model set background structure allows global regularity to result from the structure of local configurations

$$
W_{\mathcal{T}_{\Delta}^{k}}=\prod_{V T_{i j} \sqsubset \mathcal{T}_{\Delta}^{k}} W_{V T_{i j}},
$$

where the pair of vertex types $(i j)$ consider the connectivity of space-filling tilings and in the case of non-space-filling tilings we consider a ball of radius $R$ (as in Appendix A, Remark A1) with respect to the subset $\mathcal{T}^{k}$.

3- Geometric realism: the symmetry of the local weights should reflect the symmetry of the underlying geometry [20,23]. Implementing the symmetry at the level of the weight itself has the bonus that the superposition of local configurations is under the admissible geometric configurations of the tiling configuration space. To use the information of the autocorrelation function $\gamma_{\triangle}$, Eq. ( A4), a signature of the local configuration is given by the relative frequency of the local $V T$ and its hits interactions with neighbors, Eq. (6), where here we restrict to inflation windows at the same level $n=m$ and one level step from the larger inflation window on the stack

$$
W_{V T_{i j}}=F\left[f_{\Delta_{\kappa}^{l}}^{r}\left(V T^{i}\right) f_{\Delta_{\kappa}^{l}}^{r}\left(V T^{j}\right) H_{i j}^{\kappa}\right]
$$

for fixed inflation windows and where the function $F$, that could give usual Boltzmann weights, here will be taken as

$$
F[f]= \begin{cases}1, & f=0, \\ f, & \text { otherwise }\end{cases}
$$

Theorem 1 (Geometric State Sum LIDS Invariance). For fixed scale, which fixes the density, the local contribution to the weights given by $W_{i}=\sum_{j} W_{V T_{i j}}$ is invariant under LIDS transformations that preserves $V T_{i}$ and its neighbor $V T_{j}$.

PROOF. The frequencies Eq. (4) are ratios of volumes, which are scaled by the same inflation $\lambda$, so that it is invariant under LIDS. The hits overlap with all neighbors and also define a locally finite specific cluster $C_{i j}$ with window $K_{C_{i j}}$ such that hits reduce to the relative frequency of $C_{i j}$ multiplied by the constant density, $H_{i}^{\kappa}=\sum_{j} H_{i j}^{K}=\operatorname{dens}\left(\triangle_{K}^{l}\right) \frac{\operatorname{Vol}\left(K_{\kappa_{i}} \cap\left(\cup K_{\kappa_{j}}\right)\right)}{\operatorname{Vol}\left(K_{\kappa}^{l}\right)}=\operatorname{dens}\left(\triangle_{K}^{l}\right) \frac{\operatorname{Vol}\left(K_{C_{i j}}\right)}{\operatorname{Vol}\left(K_{\kappa}^{l}\right)}=$ $\operatorname{dens}\left(\triangle_{K}^{l}\right) f_{\triangle_{K}^{l}}^{r}\left(C_{i j}\right)$, which is invariant.

Theorem 2 (Geometric State Sum Global Invariance under LIDS). For fixed scale, which fixes the density, the product of local contribution to the weights given by $W_{\mathcal{T}^{k}{ }^{n}}=\prod_{i}^{\mathcal{N}} W_{i}$, with $\mathcal{N}$, here, being the number of tiles in $\mathcal{T}_{\triangle}$, is invariant under LIDS transformations that preserves $V T_{i}$ with $\mathcal{N} \rightarrow \infty$.

PROOF. Due the properties of locally finite and finite local complexity, Remark $\mathrm{A} 1, W_{\mathcal{T}^{k}}{ }^{n}$ can always be written as a product of finite number of the relative frequencies of clusters $C_{i}$ with windows $K_{C_{i}}$, where these clusters include the VTs and the ones generated by the hits $\left(C_{i j}\right)$ as in theorem 1. But in the larger $\mathcal{N}$ limit, the number of a specific cluster $C_{i}$ in the general product over a tiling, must approach its frequency so that we can write $W_{\mathcal{T}_{\Delta^{n}}}=\prod_{i}^{n^{\prime}}\left(f_{\Delta_{K}^{l}}^{r}\left(C_{i}\right)\right)^{f_{\Delta_{K}^{l}}^{r}\left(C_{i}\right) \mathcal{N}}$, with $n^{\prime}$ the number of clusters $C_{i}$ s. As the frequencies are invariants, $W_{\mathcal{T}^{k}}{ }_{\Delta^{n}}$ is invariant. 
This means that $W_{\Delta}$ is an invariant on the space of (self-similar or self-replicating) tilings recursively generated by inflations $\lambda_{\kappa_{i}}^{n}$. Due the fact that local LIDS invariance is given from a center $\kappa_{i}$, it is interesting to consider dynamics from a $\kappa_{i}$ point of view. Given a specific $\kappa_{i}$ it sets two scales, one is the inflation/deflation scale where $\kappa_{i}$ is located at some level, and another is the scale $l_{g}$ for the CPS $\mathcal{G}$. Including the scale $l_{g}$ maintains the invariance at each fixed $l_{g}$. So we assume that in $W_{\mathcal{T}_{\Delta}}, \Delta$ encodes the scale $l_{g}$.

A probability measure on the finite tiling space is given from

$$
\mu_{\mathcal{T}_{\Delta}^{k}}\left(\mathcal{T}_{\Delta}^{k}\right)=\frac{1}{W_{\mathcal{T}_{\Delta}}} W_{\mathcal{T}_{\Delta}^{k}}
$$

and the expected value of a measurable function $f: \mathcal{T}^{k} \rightarrow \mathbb{R}$ by

$$
E_{\Delta}[f]:=\mu_{\mathcal{T}_{\Delta}^{k}}(f)=\sum_{\mathcal{T}_{\Delta}^{k} \sqsubset \mathcal{T}_{\Delta}} f\left(\mathcal{T}_{\Delta}^{k}\right) \frac{1}{W_{\mathcal{T}_{\Delta}}} W_{\mathcal{T}_{\Delta}^{k}}
$$

Consider now a subset $\Delta_{K_{i}}$, of some model set $\triangle$, that contains tilings $\mathcal{T}_{\Delta}^{\kappa_{i}}$ with a specific $V T$ homothetic center $\kappa_{i}$ at the origin. We can do translations of $\kappa_{i}$ with the boundary condition that its inflation window $K_{\kappa_{i}} \subset K_{\Delta}$, with $K_{\triangle}$ at the fixed level. This generates the $\mathbb{Z}$-module $Z_{\kappa_{i}}\left(\mathcal{T}_{\Delta}^{K_{i}}\right)$ and its LTM $Z\left(\mathcal{T}_{\Delta}^{\kappa_{i}}\right)$. The geometric state sum construction applies to this subset generated by the $\mathbb{Z}$-module, $W_{\mathcal{T}_{\triangle_{i}}}$, and to simplify we can rewrite Eq. (6) to this special case

$$
H_{i j}^{\kappa_{i}}=f_{\Delta}^{a}\left(\triangle_{H_{i j}}\right)=\operatorname{dens}(\triangle) \frac{\operatorname{Vol}\left(K_{\kappa_{i j}}\right)}{\operatorname{Vol}\left(K_{\triangle}\right)} .
$$

\subsection{Dynamics}

For general model sets and tilings, a concrete determination of points and tiles requires an algorithm in the embedding space that searches for $\mathcal{L}$ points and then does the necessary tests. In this sense we have a notion of points or tiles that are already projected and are part of the parallel space (they are $\mathrm{ON}$ ) and others that not. The probabilistic measure built above allow us to define random walks on a given model set $\triangle$. Basically, we start at one tiling from the sum Eq. (7), with a specific $\kappa_{i}$ at the origin that is $\mathrm{ON}$ and we can move it around in the $Z\left(\mathcal{T}_{\Delta}^{\kappa_{i}}\right)$ according some random walk setting. After some steps $N$ we have a path in parallel space given by the set of $N$ steps elements of $Z\left(\mathcal{T}_{\Delta}^{\kappa_{i}}\right), t_{\kappa_{i}}=\left\{t_{\kappa_{i}}^{l}\right\}, l=1, \ldots, N$, which represents the $\kappa_{i} V T$ at different translations $t^{l}$. This ordered path $t_{\kappa_{i}}$ defines an auxiliary space given by a set of subsets of $\triangle$ called here animation $A_{t_{\kappa_{i}}}$

$$
A_{t_{\kappa_{i}}}^{p}=\left\{\triangle_{t_{\kappa_{i}}} \mid t_{\kappa_{i}}^{l} \in Z_{\kappa_{i}}\left(\mathcal{T}_{\triangle}^{\kappa_{i}}\right), l=1 \ldots N\right\}
$$

with cardinality $N$ and each $\triangle_{t_{i}}^{l}$ with its associated tiling $\mathcal{T}_{\Delta_{t_{K_{i}}}}$. By repeating this procedure $M$ times we generate a set of animations $A_{t_{\kappa_{i}}}=\left\{A_{t_{\kappa_{i}}}^{p} \mid p=1 \ldots M\right\}$ where $p$ is a path of the random walk given a particular instance of $t_{\kappa_{i}}$.

Now we are in position to state the fourth constraining principle for geometric state sums, as discussed in the beginning of section 3:

4- Principle of efficient language (PEL) [24-26]: This aims to implement a notion of efficiency in discrete systems, which are codes or languages. The random walks with its auxiliary animations sets $A_{t_{\kappa_{i}}}$ above can be interpreted as a form of a look-ahead algorithm (so-called look-savings-ahead algorithm in [20]) that allows us to define two computational functions that can be coupled to the geometric state sum model. First we consider the so-called hit potential, $Y$, which takes values on the natural numbers including zero, $Y: \mathcal{T}^{k} \rightarrow \mathbb{N}$. At each tiling position $\kappa_{j}$ of $\mathcal{T}_{\Delta}$, that has $\kappa_{i}$ at 
origin, the hit potential $Y_{j}$ can be defined in two ways, first by the number of animations that uses that position $\kappa_{j}$ as part of its path $p=t_{\kappa_{j}}$

$$
Y_{j}=\operatorname{card}\left(\left\{t_{\kappa_{i}} \mid \kappa_{j} \sqsubset t_{\kappa_{i}}\right\}\right) .
$$

and second, in each animation $A_{t_{\kappa_{i}}}^{p}$ by the number of steps that have that position in its set

$$
Y_{j}^{a}=\operatorname{card}\left(\left\{\triangle_{t_{\kappa_{i}}^{l}} \subset A_{t_{\kappa_{i}}}^{p} \mid \kappa_{j} \sqsubset \mathcal{T}_{\triangle_{t_{K_{i}}}}\right\}\right) .
$$

We note that a random walk distribution with values on $\mathbb{R}$ could be used.

The second function considered is called savings potential, $S: \mathcal{T}^{k} \rightarrow \mathbb{N}$. In each animation $A_{t_{K_{i}}}^{p}$ we count the number of overlaps between the path positions $t_{\kappa_{i}}$ and the union of their associated tiling inflation sets $\mathcal{T}_{\Delta_{t_{K_{i}}}}$

$$
S\left(A_{t_{\kappa_{i}}}^{p}\right)=\operatorname{card}\left(\left\{t_{\kappa_{i}} \sqcap\left(\sqcup \mathcal{T}_{\Delta_{t_{K_{i}}}}\right) \mid \triangle_{t_{\kappa_{i}}^{l}} \subset A_{t_{\kappa_{i}}}^{p}, l=1, \ldots, N\right\}\right)-N
$$

In this way we can consider the state sums coupled with the subset of animations $A_{\kappa_{i}}, W_{\mathcal{T}_{\kappa_{i}}}$,

$$
W_{\mathcal{T}_{\Delta}}=\sum_{\mathcal{T}_{\Delta}^{k} \sqsubset \mathcal{T}_{\Delta}} W_{\mathcal{T}_{\Delta}^{k}} W_{\mathcal{T}_{A_{k_{i}}}^{k}},
$$

with

$$
W_{\mathcal{T}_{A_{\kappa_{i}}}^{k}}=\sum_{A_{\kappa_{i}} \subset \mathcal{T}_{A_{\kappa_{i}}^{k}}^{k}} \prod_{V T_{j} \sqsubset A_{\kappa_{i}}} F\left[S\left(A_{\kappa_{i}}\right) f_{\triangle_{\kappa}^{l}}^{r}\left(V T_{j}\right) Y_{j}\right]
$$

with the hit potential from Eq. (15) and $W_{V T_{i j}}=F\left[f_{\Delta_{\kappa}^{l}}^{r}\left(V T_{i}\right) f_{\Delta_{K}^{l}}^{r}\left(V T_{j}\right) H_{i j}^{K_{i}} Y_{i} Y_{j}\right]$ in $W_{\mathcal{T}_{\Delta}^{k}}$.

\subsection{Expected Values}

The immediate expected value of interest is the one for the tiling state itself, $W_{\mathcal{T}_{\Delta}^{k}}$

$$
E_{\Delta}\left[W_{\mathcal{T}_{\Delta}^{k}}\right]=\frac{1}{W_{\mathcal{T}_{\Delta}}} \sum_{W_{\mathcal{T}_{\Delta}^{k}} \sqsubset W_{\mathcal{T}_{\Delta}}} W_{\mathcal{T}_{\Delta}^{k}}^{2} W_{\mathcal{T}_{A_{\kappa_{i}}}^{k}}
$$

Note that if we use Eq. (15) in Eq. (18) without the savings potential contribution, the main contribution to the weights will come from tiles, which are translations of $\kappa_{i}$. We can get a simpler expression for this expected value on the extreme situation where all the random walks steps are constrained to oscillating around the neighbors of the $\kappa_{i}$ at the origin so that the tiling contributions (on a reduced model set $\Delta^{o}$ ) reduces to the $V T_{K_{i}}$ itself and its immediate neighbors, $V T_{K_{l}}$. In this situation we can consider only the expected value of $H_{i j}^{K_{i}}$, which, to simplify the notation, we include the frequencies $H_{i j}^{K_{i}} \rightarrow f_{\Delta_{K}^{l}}^{r}\left(V T_{i}\right) f_{\Delta_{K}^{l}}^{r}\left(V T_{j}\right) H_{i j}^{K_{i}}$

$$
E_{\Delta^{o}}\left[H_{i j}^{K_{i}}\right]=\frac{\sum_{W_{\mathcal{T}_{\Delta}^{j}} \sqsubset W_{\mathcal{T}_{\Delta}}}\left(\prod_{l}\left(H_{i l}^{K_{i}}\right)^{2} Y_{i} Y_{l}\right)_{j}}{\sum_{W_{\mathcal{T}_{\Delta}^{j}} \sqsubset W_{\mathcal{T}_{\Delta}}}\left(\prod_{l} H_{i l}^{K_{i}} Y_{i} Y_{l}\right)_{j}} .
$$

which has well defined $k$-momentum

$$
E_{\Delta^{o}}\left[\left(H_{i j}^{K_{i}}\right)^{k}\right]=\frac{\sum_{W_{\mathcal{T}_{\Delta}^{j}} \sqsubset W_{\mathcal{T}_{\Delta}}}\left(\prod_{l}\left(H_{i l}^{K_{i}}\right)^{k+1} Y_{i} Y_{l}\right)_{j}}{\sum_{W_{\mathcal{T}_{\Delta}^{j}} \sqsubset W_{\mathcal{T}_{\Delta}}}\left(\prod_{l} H_{i l}^{K_{i}} Y_{i} Y_{l}\right)_{j}} .
$$


As the dependence of the hit potential on the random walk probability distribution can be made the same for any type of $\kappa_{i}$, the expected value invariant distinction between different $V T \mathrm{~s} \kappa_{i}$ is given by the absolute frequency Eq. (13), which is a LIDS invariant but contains a part $l_{g}$-invariant, the relative frequency, and a part dependent on this scale given by its density. This can be computed numerically and ratios can be established between different VTs. Later we will discuss some specific examples and implications this has for physics.

Relaxing the condition on the random walks, letting them spread over larger number of tiles and noting that the weights contributions are from the tiles, which are translations of $\kappa_{i}$, the expected value Eq. (20) gives the expected value of a pattern in the animations $A_{t_{\kappa_{i}}}^{p}$ generated by the translations of $\kappa_{i}$, which depends mainly on the savings and hit potentials. If we restrict the expected values of the hit potential, disconsidering savings contributions

$$
E_{\Delta}\left[Y_{i} Y_{j}\right]=\frac{\sum_{W_{\mathcal{T}_{\Delta}^{j}} \sqsubset W_{\mathcal{T}_{\Delta}}}\left(\prod_{l} H_{i l}^{K_{i}}\left(Y_{i} Y_{l}\right)^{2}\right)_{j}}{W_{\mathcal{T}_{\Delta}}} .
$$

which can be used to probe the possible kind of path pattern generated by the random walks by considering a improved computational accumulative hit potential function where in Eq. (15) we consider a set of $t_{\kappa_{i}}$ starting at different positions of the same $\kappa_{i}$ in $Z\left(\mathcal{T}_{\Delta}^{\kappa_{i}}\right)$.

As the geometric state sum is built from the geometry of the windows polytopes it is possible to compute expected values of geometrical quantities such as length, area and volume, $V: \mathcal{T}^{k} \rightarrow \mathbb{R}$. For example, for the volume $E_{\Delta}\left[\mathcal{V}\left(W_{\mathcal{T}_{A_{i}}}\right)\right]$, in the case analogous of Eq. (21)

$$
E_{\Delta^{o}}\left[\mathcal{V}^{\kappa_{i}}\right]=\frac{\sum_{W_{\mathcal{T}_{\Delta}^{j}} \sqsubset W_{\mathcal{T}_{\Delta}}}\left(\prod_{l}\left(\operatorname{Vol}\left(K_{\kappa_{i l}}\right)\right)^{2} Y_{i} Y_{l}\right)_{j}}{\sum_{W_{\mathcal{T}_{\Delta}^{j}} \sqsubset W_{\mathcal{T}_{\Delta}}}\left(\prod_{l} \operatorname{Vol}\left(K_{\kappa_{i l} l}\right) Y_{i} Y_{l}\right)_{j}} .
$$

The last expected value we will consider is for the autocorrelation function Eq. (A4)

$$
E_{\Delta}\left[\gamma_{\kappa_{i}}\right]=\frac{1}{W_{\mathcal{T}_{\Delta}}} \sum_{W_{\mathcal{T}_{\Delta}^{j}} \subset W_{\mathcal{T}_{\Delta}}} \gamma_{\kappa_{i}}^{j} W_{\mathcal{T}_{\Delta}^{j}} W_{\mathcal{T}_{\kappa_{i}}^{j}}
$$

where $\gamma_{\kappa_{i}}^{j}$ is the autocorrelation on the tiling $j$ with regard the translations of $\kappa_{i}$. This expected value allows to define an order parameter, hit averaging $\bar{H}$,

$$
\bar{H}_{\Delta}=\frac{E_{\Delta}\left[\gamma_{\kappa_{i}}\right]}{N},
$$

where the meaning of the order parameter can be seen by looking in $\Delta^{o}$ over the scale $l_{g}^{o}$. Under a coarse-grained process in parallel space, there is an inverse process in perpendicular space, where the higher density limit dens $\left(\Delta_{\kappa_{i}}\right) \rightarrow 1$ sets a fixed point where the state sum is also scale invariant and uniform distribution is enforced.

\subsection{Model Sets Examples}

The model sets of interest here are the ones for $d=3$. The most studied family of model sets are the ones for which the relative frequency Eq. (A1), for their VTs, are elements of the ring of integers [15], here called Dirichlet integers,

$$
\mathbb{Z}[\phi]=\{m+n \phi \mid m, n \in \mathbb{Z}\}
$$

where $\phi=\frac{\sqrt{5}+1}{2}$ is the golden ratio. This family includes the 3 -dimensional icosahedral model sets [15]. The two main examples are projected from the $Z^{6}$ lattice, $G_{Z^{6}}=\left(\mathbb{R}^{3}, \mathbb{R}^{3}, Z^{6}\right)$, and from the $D_{6}$ lattice, $\mathcal{G}_{D_{6}}=\left(\mathbb{R}^{3}, \mathbb{R}^{3}, D_{6}\right)$. The $\mathcal{G}_{Z^{6}}$ model set has $24 V T$ s and $\mathcal{G}_{D_{6}}$ has $36 V T$ s and tables with explicitly values for the relative frequencies can be found for example in [27]. 
For these two model sets the scale invariant part of $E_{\Delta^{o}}\left[H_{i j}^{K_{i}}\right]$ is an element of $\mathbb{Z}[\phi]$ as its powers are again in $\mathbb{Z}[\phi]$ due to $\phi^{2}=\phi+1$. Ratios between $E_{\Delta^{o}}\left[H_{i j}^{K_{i}}\right]$ for different $\kappa_{i}$-VTs have invariant part, which are ratios between Dirichlet integers.

The $\mathcal{G}_{Z^{6}}$ and $\mathcal{G}_{D_{6}}$ model sets are space-filling. Possible $\kappa$-inflation non-space-filling tilings in these cases can be re-scaled to space-filling ones. Their $V T$ s are not regular polytopes, but are deformed projections from hyper dimensional regular polytopes in the embedding lattice. Another example is derived from the $E_{8}$ lattice [28], which are non-space-filling tilings, so-called compound quasicrystals (CQC) or n-component model sets (see [15] for an example of a 3-component model set describing the Danzer's ABCK tiling). $E_{8}$ has a well known 4-dimensional model set implementation, the so-called Elser-Sloane model set [29], which can be naturally extended to 3-dimensional model sets [30]. The 3-dimensional $E_{8}$-CQC uses a different approach by considering 6-dimensional sub-spaces of $E_{8} \cdot \mathcal{G}_{E_{8}}^{n}=\left(\mathbb{R}^{3}, \mathbb{R}^{3}, L_{n}^{6} \subset E_{8}\right)$. One can employ the usual CPS on the 6-dimensional sub-spaces by considering the canonical window from the Voronoi polytope there, but as we don't need space-filings tilings, the spherical approximation here can be taken as the window defining the CPS. The construction relies only on one window $K$ and $\kappa$-inflations given by powers of $\phi$. We specifically consider the cut-window to be scaled by $\phi^{1}, \phi K$, and one inflation window by $\phi^{0}, K$. This construction makes it possible to combine periodic and aperiodic order by having model sets with tilings with regular $V T$ s, which are tetrahedrons or groups of tetrahedrons, where the groups considered are multiples of groups of four tetrahedra, up to $20(n 4 G, n=1,2,3,4,5)$, that can occupy the same center position $\kappa_{i}$ and generate convex hulls of crystallographic objects such as the cuboctahedron.

This procedure gives the relative frequencies for $\kappa$-inflation tilings to be elements of $\mathbb{Z}\left[\phi^{-1}\right]$, and to be the same for all $n 4 G \mathrm{~s}$. So the scale invariant part of $E_{\Delta^{o}}\left[H_{i j}^{K_{i}}\right]$ is the same for the different $n 4 G \mathrm{~s}$ and the scale dependent density, dens $\left(\triangle_{n 4 G}\right)$, being the important part. Scale invariant information for the expected values for different $n 4 G$ are given by ratios between the different densities.

In the next section we provide a novel method, so-called texture scheme (TMS), to do the explicit computations by embedding in a lattice, a set, which is isomorphic and very close to the original model set.

\subsubsection{Explicit construction of the $E_{8}-\mathrm{CQC}$}

An analytical method to build the $Z[\phi]$-related model sets and the CQC, which gives analytical foundations for the densities, has been recently developed. We'll describe the analytic construction of the model set, in lattice coordinates, with a lattice of the target space, not of the embedding space. That makes a huge dimension reduction and accelerates the computation, without any loss on information of the model set.

The union of five model sets of $\mathcal{G}_{E^{8}}^{n}$, compounded together in a unique 3-dimensional space embedded in $\mathbb{R}^{3}$, are discrete sets in $\mathbb{D}^{3}$, the cartesian product of 3 instances of the Dirichlet ring, see equation (27). Our new description of this model set considers a discrete texture of the target space embedded in a lattice ( $\mathbb{Z}^{3}$ in 3-dimensions), and a family of coordinate transformations between the most compact set and the exact model set in $\mathbb{D}$ coordinates.

Using the same notation of section 2 we define a TMS as a 3-tuplet $\mathcal{G}^{\prime}=\left(\mathbb{Z}^{d}, G^{\prime}, \mathcal{L}\right)$, where $\mathbb{Z}^{d}$ is a euclidean cubic lattice, $G^{\prime}$ is a bounded region of a nonlinear transformation of $\mathbb{Z}^{d}$ into a unit cube and $\mathcal{L}$ is a lattice in $\mathbb{Z}^{d} \times G^{\prime}$, with the two natural projections $\pi: \mathbb{Z}^{d} \times G^{\prime} \rightarrow \mathbb{Z}^{d}$ and $\pi_{\perp}: \mathbb{Z}^{d} \times G^{\prime} \rightarrow G^{\prime}$. With $L=\pi(\mathcal{L}), \pi$ is a bijection between $\mathcal{L}$ and $L$, and $\pi_{\perp}(\mathcal{L})$ dense in $G^{\prime}$. $\mathcal{E}=\mathbb{Z}^{d} \times G^{\prime}$ is again the embedding space, the space $\mathbb{Z}^{d}$ is the parallel space and $G^{\prime}$ is the virtual perpendicular or virtual internal space.

This scheme has also the well-defined star map applied to $G^{\prime}, \star: L \rightarrow G^{\prime}$ :

$$
\star(x)=x / \phi-\lfloor x / \phi\rceil .
$$

The star cube, $\star\left(\mathbb{Z}^{3}\right)$, a cube of unit edge length, centered at point $O=\{0,0,0\}$. The virtual spherical cut window is inside of this cube but has a smaller diameter $\phi^{-1}$ and is centered on $\kappa_{i}$. Restricting to points, which are centroids of a specific $V T$, corresponds to operating on a subset of the star cube. For the $V T$ of a group of 4 tetrahedrons, whose convex hull is a cuboctahedron, the 
restriction in the star cube is simply a smaller $O$-centred star cube of diameter $3 \phi^{-7}$. We'll define in equation (A20) a function $f_{V}(k)$ so that all model set vertices are in $f_{V}(\mathbb{Z})^{3}$ and $2|f(\mathbb{Z})|<\phi^{-1}$, and a function $f_{4 G}(k)$ so that all $4 \mathrm{G}$-centroids are in $f_{4 G}(\mathbb{Z})^{3}$ and $2\left|f_{4 G}(\mathbb{Z})\right|<3 \phi^{-7}$.

We can recover the group manifold of $G^{\prime}$ as a real $S U(2)$ manifold isomorphic to the sphere $S^{3}$. Parametrizing $S^{3}$ by the three pure imaginary quaternions $i, j, k, q=e^{2 \pi(a i+b j+c k)}$ spans $S^{3}$ when $a, b, c$ spans $\left(-\frac{1}{2},+\frac{1}{2}\right]$ and $q \bar{q}=1$ so with $a=\star(x), b=\star(y), c=\star(z)$ we use the star map to get coordinates in the Lie algebra $\mathfrak{s u}(2)$, and the exponential map toward the Lie group manifold $S^{3}$.

More details on the CQC implementation, such as how to get the precise coordinates, are shown in Appendix B.

The relative frequencies for $\kappa$-inflation tilings are computed from the distance in the perpendicular space between the star maps of two homothetic centers, $\kappa_{i}$ and $\kappa_{j}$, whose square is a Dirichlet integer.

$$
d^{2}\left(\star\left(\kappa_{i}\right), \star\left(\kappa_{j}\right)\right)=\left(\overrightarrow{\star\left(\kappa_{i}\right)}-\overrightarrow{\star\left(\kappa_{j}\right)}\right)^{2}
$$

The relative volume of the intersection of two balls of radius 1 , whose centers are distant by $d$, with respect to the enclosing ball of radius $\phi$ is (see Eq. (6)

$$
\frac{\operatorname{Vol}\left(K_{\kappa_{i j}}\right)}{\operatorname{Vol}(K)}=\frac{1}{16 \phi^{3}}(2-d)^{2}(4+d) .
$$

If $d=m+n \phi$ the equation (30) reduces to

$$
\begin{array}{r}
\frac{\operatorname{Vol}\left(K_{\kappa_{i j}}\right)}{\operatorname{Vol}(K)}=(2 \phi-3)+\frac{3}{4}((3 m-2 n)+\phi(-2 m+n)) \\
+\frac{1}{16}\left(\left(-3 m^{3}+6 m^{2} n-3 m n^{2}+n^{3}\right)+\phi m\left(2 m^{2}-3 m n+3 n^{2}\right)\right) .
\end{array}
$$

When $i=j$ we have the relative frequency of the inflation window given by $(2 \phi-3)=\phi^{-3}$, which is the volume of a ball of radius one relative to a ball of radius $\phi$. For $|d|<2 / \phi, \frac{\operatorname{Vol}\left(K_{\kappa_{i j}}\right)}{\operatorname{Vol}(K)}<$ $\phi^{-3}(1-2 \phi d / 5)$ which is a good linear approximation.

\section{Implications}

In physical terms, the quasicrystal structures described by the higher dimensional model sets mathematics discussed here are positioned at large scales or low energy, such as the quasicrystal materials built upon fundamental particles over the geometry of spacetime. The agreement of the model set theory with experiments involving quasicrystals materials is remarkable. For example, the free energy among other observables are shown to be invariant under translation of the window in perpendicular space, which gives a geometric understanding of the so-called phasons modes [19]. Here we'll discuss implications to push the model sets structures to short distances, such as the Planck scale, as a candidate for the unification structure for quantum gravity and particle physics. The motivation for this proposal comes from different fronts, such as a renewed interest in discrete mathematics with regards to the quantum gravity regime as discussed in the introduction, the problem of time, and the fact that aperiodic structures appear in solutions of quantum cosmology both with general relativity and modified gravity theories [31] (and references therein). In this inverted case we need to consider the emergence of "fundamental" particles, spacetime and their governing physical laws.

The approach of entropic or emergent gravity [1,2] considers a particle of mass $m$ approaching an holographic screen from the side where spacetime has already emerged. The holographic principle is applied to assume the encoding of the microscopic configurations, $N_{c}$, behind the screen, in the area, $A_{s}$, of the screen, $N_{c} \propto A_{s}$, and the law of inertia, the equivalence principle, Newton's gravitational law, or Einstein equations can be derived. In this paradigm, gravity is an emergent entropic force from the underlying configuration of quantum matter. In this case, one transfers the problem of emergence of spacetime to the problem of emergence of quantum matter. Once one has mass and energy distributions and its dynamics, gravity will emerge. But, in a circular way, the 
configuration of matter should be affected by gravity itself. Essentially, the holographic principle and the associated covariant entropy bound are linked to regimes where spacetime can be classically well approximated with sufficient matter content. Nevertheless the bound linked to the area and not to the volume of the region, gives a strong evidence in favors of unitarity of the underlying quantum field theory rather than locality of quantum fields as quantum evolution preserve information. It is noteworthy that the locality principle in section 3 refers to the state sum weights of the underlying model sets structures, which is about the local topology given from those structures. We will require in the construction below emergent quantum evolution unitarity without need to refer to emergent locality in a relational description.

So, how does one define evolution without a prior notion of spacetime, regarding gravity as the geometry of spacetime? The problem is having an unification of general relativity with quantum mechanics in a theory of quantum gravity, usually referred to as the problem of time in quantum gravity [32-35]. One approach, called internal time, is to consider relational evolution where some physical degree of freedom evolves relative to others, with the dynamics governed by more general Hamiltonian constraints [36]. This works well if the degree of freedom playing the role of internal time behaves monotonically, which is generally not the case. The physical system internal time can be a quantum field or some underlying structure, such as the model set discussed here. In this case it is possible to have the internal time given from a physical (a)periodic clock with turning points and with possible self-interactions. In general the choice of internal time or clocks is local and dynamic, which is the same problem of gauge fixing in quantization of gauge theories $[21,37,38]$, which leads to the conclusion that the problem of time is a special case of the so-called Gribov problem in general gauge theories. In other terms, how does one define global evolution with respect to some underlying oscillating clock system with turning points? One simple model system solution was given recently [21], where one distinguishes three necessary notions of time, the usual background time parameter, the global monotonic relativistic one and a new notion of a clock variable (more details on physical implementations recently developed on this in [22,39].) One of the implications of having the model set as the underlying structure is that it can play the role of the underlying clock, providing the global monotonic time and the clock variable, which is understood as internal time.

\subsection{Fundamental Clocks and an Emergent Observational Screen}

If there is an underlying discrete structure as the proposed model sets, one question is how matter and time emerge from those structures. To propose one possibility on the emergence of matter, let's consider that there is a fundamental limit on observation on small scales, which here translate to the coarse-grained model set scaling. Depending on each system this limit define the fundamental properties that can be observed, an emergent observational screen, which encodes all information behind it, in lower scales, so that we have access only to expected values with respect to the underlying model sets structures. From the point of view of the state sum probabilistic measure over re-scaling that limit is the higher density scale invariant limit, $\bar{H}_{\triangle^{o}} \rightarrow 1$ where the measure is locally invariant under LIDS and under the model set $l_{g}$ re-scaling; and information of expected values are available. This limit can be achieved by considering, $K_{K}^{l} \gg K_{\kappa_{i}}^{n} \cap K_{\kappa_{j}}^{m}$, in Eq. (6). Interestingly, in this limit, coarse-grained can be followed by re-scaling of $V T \mathrm{~s}$, given a notion of 2-dimensional layers spread in 3-dimensions so that the emergent observational screen has a local 3-dimensional aspect where information of both emerged and non-emerged sides can coexist.

In usual model sets relative frequencies are uniformly distributed, so one expects to have ergodicity $[15,40]$, but here the savings and the hit potentials allow for dynamics that steer probabilities away from uniform distributions, which can be interpreted as the PEL. Another aspect that can leads away from ergodicity is the more general possibility of less homogeneous non-space-filling tilings. In the regime of higher density in perpendicular space according the order parameter Eq. (26), one can expect to recover the usual equilibrium situation with a valid measure given by an usual path integral measure, $\mu_{P I}$. Similar behavior occurs with polymer physics, which can be modeled by random chains that converge to the path integral of a free particle or with ensembles of polymers described by fluctuating quantum fields [41] in the limit of large number of nodes in the chain. Therefore we can assume that expected value of functions behave on the emergent observational 
screen similarly as measured by the two different measures, $\mu_{\mathcal{T}_{\Delta}^{k}}(f)$ and $\mu_{P I}(f)$. The following function captures the emergent observational screen information

$$
f_{\Delta}^{h}[f]=\delta\left(\mu_{\mathcal{T}_{\Delta}^{k}}(f)-\mu_{P I}(f)\right),
$$

where $\mu_{P I}$ is given for the measure for usual path integral, implementing physics laws such as momentum and energy conservation, and $\delta$, the delta function. The function Eq. (32) allows us to establish a correspondence between quantities in both sides of the screen. Due theorems 1 and 2 , frequencies of clusters in the model set, are natural conserved quantities under re-scaling to be mapped to physical quantities such as mass and energy. In polymer physics it is possible to establish the correspondence of the underlying parameters of the random chains with the path integral properties, such as the link's length of the chain corresponding to the particle mass. We expect the same here, with the expected values of properties of $\kappa_{i}$ given the necessary quantum numbers of fields excitation on the emergent path integral, especially $H_{i j}^{\kappa_{i}}$ playing the role of energy in correlation function of fields. Another opportunity is to have the screen working as a background for relational evolution of the emergent degrees of freedom. Let us see how this can work.

In the limit of higher density - uniform distribution, the state sum works as a partition function, which, from the proof of theorem 2 , reduces to a product of cluster frequencies $\prod_{i}^{n^{\prime}} f_{i}^{f_{i} \mathcal{N}}$, which has the exponential asymptotic $e^{N\left(\lambda^{n}\right)\left(\sum_{i} f_{i} \ln \left(f_{i}\right)\right)}$, where the number of tiles grows with the difference of levels between inflations of the large window compared to the small ones and the new quantity that appears in the exponential we identify with the information entropy of the tiling state

$$
I_{\mathcal{T}_{k}}=-\sum_{i} f_{i} \ln \left(f_{i}\right)
$$

Then thermodynamic densities can be well defined such as the free energy $F_{E}=\lim _{N \rightarrow \infty} \frac{1}{\lambda^{n} N} \ln W_{\Delta}\left(\lambda^{n}\right)$, which is also function of clusters frequencies or hits. In terms of the partition functions from Eq. ( 32)

$$
\sum_{\mathcal{T}^{k}} e^{-N\left(\lambda^{n}\right) I_{\mathcal{T}^{k}}}-\sum_{i} e^{-\beta E_{i}}=0
$$

which allows us to establish a correspondence between tiling states probabilities and emergent energy states probabilities with $N\left(\lambda^{n}\right)$ corresponding to inverse temperature and cluster frequencies (or their information entropy) with energy.

Now that we have a notion of energy in the emergent observational screen (this is similar to the notion of a holographic screen, but without spacetime metrics, as there is an averaging over underlying degree of freedom), we can consider relational evolution by coupling in the emergent side an emergent Hamiltonian to describe additional degrees of freedom such as momentum and position of modes of some emerged quantum field, with the screen providing an effective potential for them. But the clusters frequencies or hits are in general aperiodic oscillating functions of position on parallel space $\left(f_{i}(x)\right.$ with $x$ as in Eq. (2)) as seen on the diffraction spectrum of the autocorrelation function Eq. (A5). Quantum relational evolution with respect this kind of background can be problematic even with certain simple relativistic Hamiltonians. Nevertheless an example of Hamiltonian constraint for relativistic systems working with periodic clock is given in [22] and in our notation given by

$$
C=-p_{f_{i}}^{2}-\left(\lambda^{n}\right)^{2} f_{i}^{2}+H(x, p)^{2}=0
$$

for general Hamiltonian $H(x, p)$, with $p_{f_{i}}$ being the momentum conjugated of our hits $f_{i}$ and the scaling factor given by the inflation parameter here $\left(\lambda^{n}\right)$. The simpler form for $f_{i}$ is given by the star map $f_{i}(x)=\left|x^{\star}\right|$ as hits depend on distances. Using the texture scheme periodic star map Eq. (28) we have the same fundamental clock studied in [22], which provides well defined quantum evolution for very small or very large inflations $\lambda^{n}$ compared to the energy scale.

\subsubsection{Relational Evolution and Model Sets}

As discussed in section 3.3.1, with the proposed texture scheme we can encode aperiodic information, such as the example of Appendix C, in a periodic structure (see also [42]) such as 
the perpendicular space information of the star map Eq. (28), which for working with distances in parallel space and with some normalization we rewrite as

$$
\star(x)=(|(4 x-T) / T-4\lfloor(x+T / 4) / T\rceil|-1) f^{t},
$$

where $f^{t}$ will give the turning points of the clock, and the form of this star map is shown in Figure 1. If we define relational evolution, Eq. (35), for a clock, $f_{i}$, given by Eq. (36), $f_{i}(x)=\star(x)$

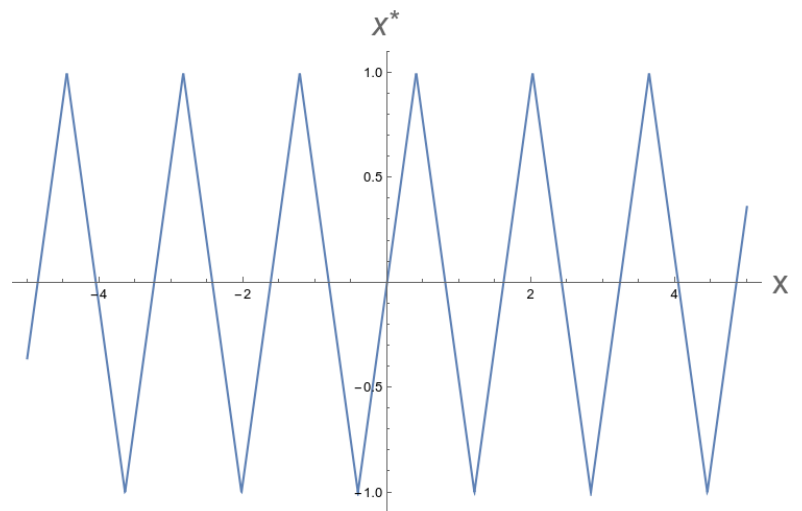

Figure 1. Periodic star map for $T=\phi$ and $f^{t}=1$.

the solution is given in [22], noting that this $f_{i}$ can be decomposed in a continuous and piecewise linear fashion so that to have $d f_{i} / d x= \pm 1$, the only difference being the irrational period, which we keep as an additional parameter $T$. For relational evolution with regard the degree of freedom $f_{i}$, the Hamiltonian constraint problem reduces to compute evolving wave functions or transition amplitudes by solving ordinary differential equations in the energy representation, where $E_{k}$ is one of the energy eigenvalues of $\hat{H}$ and $\psi_{k}$ the corresponding eigenfunction (omitting the level $n$ of $\lambda$ inflation)

$$
i \hbar \frac{\mathrm{d} \psi_{k}\left(f_{i}\right)}{\mathrm{d} f_{i}}= \pm \sqrt{E_{k}^{2}-\lambda^{2} f_{i}^{2}} \psi_{k}\left(f_{i}\right),
$$

which has turning points on $f_{i}^{t}=E_{k} / \lambda$. The solution is given, unwinding the time clock by replacing $f_{i}(x)=\star(x)$ explicitly, in terms of $x$ by

$$
\psi_{k}(x)=\psi_{k}\left(x_{0}\right) e^{\left(i \operatorname{sgn}\left(\mathrm{d} f_{i} / \mathrm{d} x\right)\left(\Theta_{k}\left(f_{i}(x)\right)-\Theta_{k}\left(f_{i}\left(x_{0}\right)\right)\right)\right)}
$$

with some initial $f_{i}^{0}\left(x_{o}\right)$ in a given half-cycle of the clock, and with the phase

$$
\Theta_{k}\left(f_{i}\right)=-\frac{1}{2 \hbar}\left(f_{i} \sqrt{E_{k}^{2}-\lambda^{2} f_{i}^{2}}+\frac{E_{k}^{2}}{\lambda} \arcsin \left(\frac{\lambda f_{i}}{E_{k}}\right)\right) .
$$

Following [22] evolution for the ground state of a harmonic system Hamiltonian can be then evaluated for different regimes of $\lambda$ with a lack of smoothness but preserving unitarity and continuity. With very small $\lambda$, the usual quantum mechanics evolution with a monotonic time parameter is trivially recovered. For intermediary values a finite decoherence time appears, but for large $\lambda$ $\left(\lambda^{n} \rightarrow \infty, f^{t} \rightarrow 0\right)$ where the period of the clock, $T_{\mathrm{C}}=T f^{t}=T E_{k} / \lambda$ is much smaller than the emerged quantum system period, $T_{\mathrm{S}}=2 \pi \hbar / E_{k}$, usual evolution is again recovered

$$
\psi_{k}(\tau) \approx \psi_{k}(0) \exp \left(-\frac{i \pi E_{k}}{T \hbar} \tau\right)
$$

where we wrote the parallel space distance $x=\tau$ to emphasise its role as the relativistic time. One difference from usual quantum evolution is a re-scaling of the system period by a factor of $T / \pi$, which for periodic clock would make sense to have only one clock period $T$, which then can be absorbed in a bare frequency $\omega^{\prime}=T \omega / \pi$ that appears in the expression of the emergent system Hamiltonian. But in the case of model sets, even if we focus only in one specific CPS, there is the 
natural possibility to have two or more fundamental periods by splitting the CPS for different $V T$ s and its $\mathbb{Z}$-module. This is easily seen for Eq. (33), where ignoring hits reduces the $V T$ frequencies, which we compute for $\mathcal{G}_{Z^{6}}$ and is shown in Figure 2. Including hits, they are ratios of volumes of

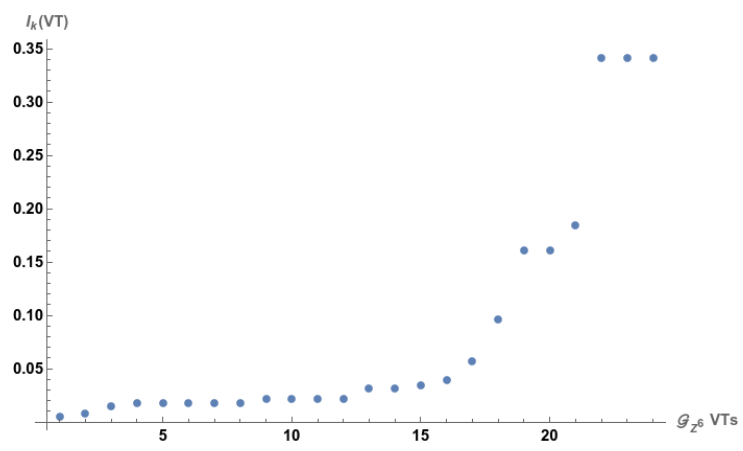

Figure 2. $I_{k}$ for each of the $24 V T$ s of $\mathcal{G}_{Z^{6}}$.

associated cluster's windows over a fixed larger window. Using the spherical approximation for the windows and considering that for the model sets of interest the radius is a number in $\mathbb{Z}\left[\phi^{-1}\right]$ or $\mathbb{Z}[\phi]$, which can be written as $\left(m+n \phi^{p}\right)$ where $m, n \in \mathbb{N}$ and $p \in \mathbb{Z}$. The general form of Eq. (33) is invariant over the variations of the parameters and is shown in Figure 3 where, without normalization, it has a maximum in $e^{-1}$ but with most states in lower scale away from this maximum and with visible gaps, resembling the rest mass spectrum of known particles including the composite hadrons. A zoom in a small scale is shown in Figure 4. The requirement of the multiple periods $T$

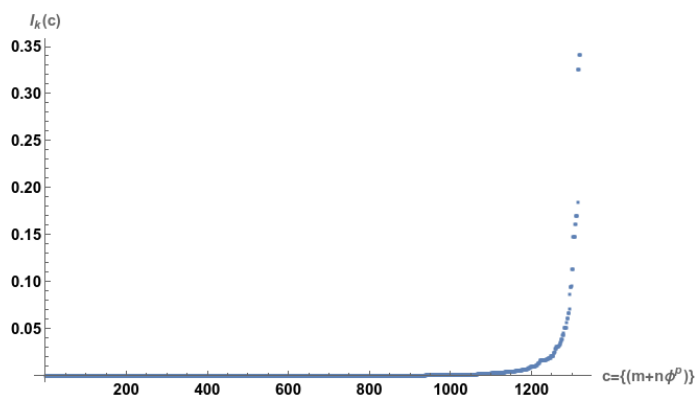

Figure 3. Sorted $I_{k}$ for different windows with radius $\left(m+n \phi^{p}\right)$ in a large window $\left(m_{\max }+n_{\max } \phi^{p_{\max }}\right)$.

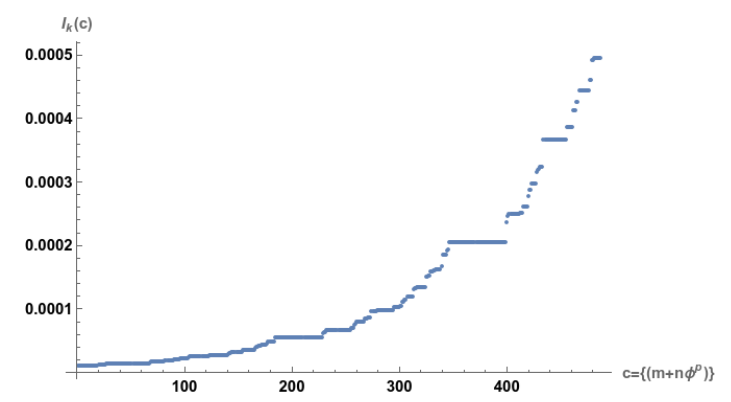

Figure 4. Zoom in a intermediary scale of sorted $I_{k}$ for different windows with radius $\left(m+n \phi^{p}\right)$ in a large window $\left(m_{\max }+n_{\max } \phi^{p_{\max }}\right)$.

is clear in the examples of Appendix $\mathrm{C}$, and also can be seen with our periodic star map where each $n 4 G$ kind of $V T$ of the $E_{8}$-CQC non-space-filling model sets defines specific tilings with specific inner distances, which we can re-scale to have all $n 4 G$ tilings with the same distances, but them including the scale factor, in each case, to affect the period of the star map. In the large density limit this doesn't matter but as we are seeing, to recover quantum evolution the clock needs to be many orders of magnitude below the quantum system oscillations, justifying the role of different small $T$. This emphasises the reflexively self-generating, self-modeling, self-contained nature of a emergent observational screen where the underlying structure must provide properties such as 
mass for the emergent quantum system while at the same time playing the role of the clock for relational evolution. This means that each fundamental bound-state system, such as the hadrons, has its own associated clock, which provides its mass and relational evolution. In this case, given a CPS, with its set of disjoint tilings $\mathcal{T}_{k}$ given frequencies and associated different $I_{k}$ and $T_{k}$, the hadronic spectrum, $\omega_{h}$, could be derived by fixing one reference frequency $\omega, \omega_{h}=\frac{\pi}{T_{k}} \omega$, consistent with the thermodynamic correspondence Eq. (34), $\omega_{h} \sim I_{k}$. Considering explicit hadronic states [43] with $E_{n l} \sim M_{h}^{\frac{3}{2}}\left(2 n+l-\frac{1}{2}\right)^{\frac{2}{3}}$ gives a direct map with hadronic masses $M_{h}$. This allows to have a slight different prediction, from [22,39], of the upper bound on the fundamental clock frequency as it depends on the quantum system of consideration predicting different bounds for different fundamental systems.

In summary, the measure $\mu_{\mathcal{T}^{k}}$ plays the role of a generator of mass and a clock for relational evolution (note that it is similar to the role of fast variables in [14] or the neural network variables in $[44,45])$. Model sets provide one way to reconcile discrete, finite systems with invariance under continuous symmetry groups, addressing the problem "How can discrete, finite systems such as fields on a lattice, display invariance under continuous symmetry groups?" [46]. The novel possibility arises in terms of spacetime symmetry by realizing that the discrete and continuous can coexist over LIDS transformation leading to the notion of a emergent observational screen discussed above and in terms of charge gauge symmetry by generalizing the group $G$ in the CPS $\mathcal{G}$ to more general groups such as the Lie groups necessary for the standard model of particle physics, $S U(3), S U(2)$ and $U(1)$, and interpreting the perpendicular space, as also generating charge in addition of mass and time.

Acknowledgments: We thank and acknowledge Dugan Hammock for providing the base code used to test some of the concepts discussed in this paper, such as the CQC model set derived from $E_{8}$.

\section{Appendix A. Review of Model Sets}

Some properties are representative of model sets $\Delta$ and for completeness we review them, following mainly [15]. This is based on measure theory background [47], which means that we have a probabilistic space as the underlying structure.

\section{Appendix A.1. Model Sets Properties}

Remark A1 (Model sets properties). 1. Uniformly discrete: There is radii $r>0$ so that each ball of radius $r$ contains at most one point of $\triangle$;

2. Relatively dense: There is radii $R>0$ so that each ball of radius $R$ contains at lest one point of $\triangle$;

3. Locally finite: For all compact $\Lambda \subset \mathbb{R}^{d}$, the intersection $C=\Lambda \cap \Delta$ (called cluster) is a finite or empty set;

4. Finite local complexity (FLC): The collection $\left\{(t+\Lambda) \cap \Delta \mid t \in \mathbb{R}^{d}\right\}$, usually represented by translations $\triangle-\triangle$, contains only finitely clusters up to translations.

5. Uniform distribution: A theorem proved in [15], which says that given some ordering sequence $\left(x_{i}\right)_{i \in \mathbb{N}}$ of the points on $\triangle$, the sequence $\left(x_{i}^{\star}\right)_{i \in \mathbb{N}}$ is uniformly distributed in $K$.

Point sets with properties 1 and 2 above are called Delone Sets. A characteristic property of a point set in $\mathbb{R}^{d}$ is the average number of points per unit volume. We are interested in the relative frequency $f^{r}$ and absolute frequency $f^{a}$ of sub-sets or clusters of a model set. Due to uniform distribution property the relative frequency of a cluster can be computed as ratios of windows volumes

$$
f_{\Delta}^{r}(C)=\frac{\operatorname{Vol}\left(K_{C}\right)}{\operatorname{Vol}(K)},
$$


where $K_{C}$ is the compact window or coding region of the $C \subset \triangle$, given by $K_{C}:=\cap_{x \in C}\left(K-x^{\star}\right)$, and $\mathrm{Vol}$ gives the volume of the windows. The absolute frequency is given by

$$
f_{\Delta}^{a}(C)=\operatorname{dens}(\triangle) f_{\Delta}^{r}(C)
$$

where $\operatorname{dens}(\triangle)$ is the density of the model set

$$
\operatorname{dens}(\Delta)=\lim _{r \rightarrow \infty} \frac{\operatorname{card}\left(\Delta_{r}\right)}{\operatorname{Vol}\left(B_{r}(0)\right)},
$$

where for model sets the limit inferior and superior coincide. The open ball of radius $r$ around $x$ is given by $B_{r}(x)$, and card is the set cardinality.

A hallmark function widely used to characterize a model set is the autocorrelation function

$$
\gamma_{\Delta}=\sum_{t \in \Delta-\Delta} \operatorname{dens}(\Delta) \frac{\operatorname{Vol}\left(K \cap\left(K-t^{\star}\right)\right)}{\operatorname{Vol}(K)} \delta_{t},
$$

from where the diffraction spectrum can be computed from the Fourier transform

$$
\widehat{\gamma}_{\triangle}=\sum_{k \in \bar{L}} I(k) \delta_{k}
$$

with $\bar{L}=\pi(\overline{\mathcal{L}}), \overline{\mathcal{L}}$ the dual lattice of $\mathcal{L}$, and the coefficients $I(k)=|A(k)|^{2}$, which in the case of internal space $\mathbb{R}^{d}$ is given by

$$
A(k)=\frac{\operatorname{dens}(\Delta)}{\operatorname{Vol}(K)} \int_{K} e^{2 \pi i\left(y k^{\star}\right)} d y .
$$

In practical application it is useful to use the spherical approximation of the window considering a ball $B_{R_{w}}=B_{R_{w}}(0)$ of radius $R_{w}$, in spherical coordinates

$$
A(k)=\frac{\operatorname{dens}(\triangle)}{\operatorname{Vol}\left(B_{R_{w}}\right)} \int_{B_{R_{w}}} e^{2 \pi i\left(y k^{\star}\right)} d y=\operatorname{dens}(\triangle) \frac{\Gamma\left(\frac{d}{2}+1\right)}{\left(\left|k^{\star}\right| \pi R_{w}\right)^{\frac{d}{2}}} J_{\frac{d}{2}}\left(2 \pi\left|k^{\star}\right| R_{w}\right),
$$

where $\Gamma$ is the gamma function, $J$ the Bessel functions of the first kind and the radius for an approximating spherical window given by

$$
R_{w}=\left(\frac{\operatorname{vol}(K)}{\pi^{\frac{d}{2}}} \Gamma\left(\frac{d}{2}+1\right)\right)^{\frac{1}{d}}
$$

\section{Appendix A.2. Model Sets Patterns and Tilings}

A pattern $\mathcal{T}$ in $\mathbb{R}^{d}\left(\mathcal{T} \sqsubset \mathbb{R}^{d}\right)$ is a non-empty set of non-empty subsets of $\mathbb{R}^{d}$. The elements of $\mathcal{T}$ are the fragments of the pattern $\mathcal{T}$. A locally finite point set such as $\triangle$ is naturally turned into a pattern as

$$
\mathcal{T}=\mathcal{T}_{\Delta}=\{\{x\} \mid x \in \Delta\},
$$

A tiling in $\mathbb{R}^{d}$ is a pattern $\mathcal{T}=\left\{T_{i} \mid i \in I\right\} \sqsubset \mathbb{R}^{d}$, where $I$ is a countable index set, the fragments $T_{i}$ of $\mathcal{T}$ are non-empty closed sets in $\mathbb{R}^{d}$ subject to the conditions

Remark A2 (Tiling conditions). 1. $\cup_{i \in I} T_{i}=\mathbb{R}^{d}$,

2. $\quad \operatorname{int}\left(T_{i}\right) \cap \operatorname{int}\left(T_{j}\right)=\varnothing$ for all $i \neq j$ and

3. $T_{i}$ being compact and equal to the closure of its interior $T_{i}=\overline{\operatorname{int}\left(T_{i}\right)}$.

If we release condition 1 above we have a non-space filing tiling or pattern. The $T_{i}$ are called regular tiles of the tiling and their equivalence class up to congruence are called prototiles. The 
locally finite property of the point set extends naturally to the pattern $\mathcal{T}$ and also the definition of a cluster $C=\Lambda \sqcap \mathcal{T}$ of $\mathcal{T}$.

Two locally finite patterns $\mathcal{T}$ and $\mathcal{T}^{\prime}$ are locally indistinguishable $(\mathrm{LI}), \mathcal{T} \stackrel{l i}{\sim} \mathcal{T}^{\prime}$, when any cluster of $\mathcal{T}$ occurs also in $\mathcal{T}^{\prime}$ and vice versa. This means that there are translations $t, t^{\prime} \in \mathbb{R}^{d}$ such that $\mathcal{T} \sqcap \Lambda=\left(-t^{\prime}+\mathcal{T}^{\prime}\right) \sqcap \Lambda$ and $\mathcal{T}^{\prime} \sqcap \Lambda=(-t+\mathcal{T}) \sqcap \Lambda$. The translation of a tiling is understood as $t+\mathcal{T}=\left\{t+T_{i} \mid i \in I\right\}$. Locally indistinguishability is an equivalence on the class of patterns, written as $L I(\mathcal{T})$.

A $\mathbb{Z}$-module for a given $\Lambda$ and $\mathcal{T}, Z_{\Lambda}(\mathcal{T})$, for some $x \in \mathbb{R}^{d}$, is defined as

$$
Z_{\Lambda}(\mathcal{T})=<t \mid \mathcal{T} \sqcap(x+\Lambda)=(-t+\mathcal{T}) \sqcap(x+\Lambda)>_{\mathbb{Z}},
$$

which is the $\mathbb{Z}$-module generated by all translations between occurrences of some $\Lambda$-cluster in $\mathcal{T}$. When $\Lambda \subset \Lambda^{\prime}$, one has $Z_{\Lambda^{\prime}}(\mathcal{T}) \subset Z_{\Lambda}(\mathcal{T})$ and $Z_{\Lambda \cup \Lambda^{\prime}}(\mathcal{T})=Z_{\Lambda}(\mathcal{T}) \cap Z_{\Lambda^{\prime}}(\mathcal{T})$. The limit translation module (LTM) $Z(\mathcal{T})$ is then defined as the inductive limit of the $Z_{\Lambda}(\mathcal{T})$ over all $\Lambda \subset \mathbb{R}^{d}$, ordered according to inclusion. The $Z(\mathcal{T})$ is an invariant of $L I(\mathcal{T})$.

A pattern $\mathcal{T}^{\prime} \sqsubset \mathbb{R}^{d}$ is locally derivable (LD) from a pattern $\mathcal{T}, \mathcal{T} \stackrel{l d}{\sim} \mathcal{T}^{\prime}$, when a compact neighborhood $\Lambda \subset \mathbb{R}^{d}$ of 0 exists such that whenever $(-x+\mathcal{T}) \sqcap \Lambda=(-y+\mathcal{T}) \sqcap \Lambda$ holds for $x, y \in \mathbb{R}^{d}$, one also has $\left(-x+\mathcal{T}^{\prime}\right) \sqcap\{0\}=\left(-y+\mathcal{T}^{\prime}\right) \sqcap\{0\}$. This extends to LI classes of patterns. A class $L I\left(\mathcal{T}^{\prime}\right)$ is called $\operatorname{LD}$ from $L I(\mathcal{T}), L I(\mathcal{T}) \stackrel{l d}{\sim} L I\left(\mathcal{T}^{\prime}\right)$, when patterns $\mathcal{T}_{1} \in L I(\mathcal{T})$ and $\mathcal{T}_{1}^{\prime} \in L I\left(\mathcal{T}^{\prime}\right)$ exists such that $\mathcal{T}_{1} \stackrel{l d}{\sim} \mathcal{T}_{1}^{\prime}$. And also apply to the point set itself: two model sets obtained from the same CPS, but with different windows $K_{1}$ and $K_{2}$, satisfy $\triangle\left(K_{1}\right) \stackrel{l d}{\sim} \Delta\left(K_{2}\right)$ if and only if $K_{2}$ can be expressed as a finite union of sets each of which is a finite intersection of translates of $K_{1}$, with translations from $L^{\star}$.

Two patterns $\mathcal{T}_{1}$ and $\mathcal{T}_{2}$ (similarly for two LI classes) are called mutually locally derivable (MLD) from each other when $\mathcal{T}_{1} \stackrel{l d}{\sim} \mathcal{T}_{2}$ and $\mathcal{T}_{2} \stackrel{l d}{\sim} \mathcal{T}_{1}$. MLD is an equivalence relation on patterns (or LI classes), $\mathcal{T}_{1} \stackrel{\text { mld }}{\sim} \mathcal{T}_{2}$. It is straightforward to show that with $\mathcal{T}^{l d} \sim \mathcal{T}^{\prime}$ one has $Z(\mathcal{T}) \subset Z\left(\mathcal{T}^{\prime}\right)$ and with $\mathcal{T}_{\sim}^{\text {mld }} \mathcal{T}^{\prime}$ one has $Z(\mathcal{T})=Z\left(\mathcal{T}^{\prime}\right)$. Then the LTM $Z(\mathcal{T})$ of $\mathcal{T}$ is an invariant of the entire MLD class of $L I(\mathcal{T})$.

A pattern $\mathcal{T}$ is translationally repetitive when, for every compact $\Lambda$ there is a compact $\Lambda^{\prime} \subset \mathbb{R}^{d}$ such that for every $x, y \in \mathbb{R}^{d}$, the relation $\mathcal{T} \sqcap(x+\Lambda)=(-t+\mathcal{T}) \sqcap(y+\Lambda)$ holds for some $t \in \Lambda^{\prime}$. The set $\Lambda^{\prime}$ quantifies the local search space to locate arbitrary $\Lambda$-clusters of $\mathcal{T}$. For $\mathcal{T} \stackrel{l d}{\sim} \mathcal{T}^{\prime}$, of $\mathcal{T}$ is repetitive, then so is $\mathcal{T}^{\prime}$.

The local topology is defined for two FLC sets $\Delta, \Delta^{\prime}$ so that they are $\varepsilon$-close when one has $\Delta \cap B_{1 / \varepsilon}(0)=\left(-t+\Delta^{\prime}\right) \cap B_{1 / \varepsilon}(0)$ for some $t \in B_{\varepsilon}(0)$. The topology is generated by the possible neighborhoods with all $\varepsilon>0$ sufficiently small and a metric topology. This topology permits the concept of a continuous geometric hull $X(\Delta)$ given by $X(\Delta)=\left\{\overline{t+\Delta \mid t \in \mathbb{R}^{d}}\right\}$, and we have that $X(\Delta)=L I(\Delta)$ and $\Delta$ is repetitive. The discrete hull, $X_{0}(\Delta)$, is given by $X_{0}(\Delta)=$ $\left\{\Delta^{\prime} \in X(\Delta) \mid 0 \in \Delta^{\prime}\right\}$.

\section{Appendix A.3. Model Sets Inflation-Deflation Symmetry}

To go beyond the "classic" symmetries, one needs an extension of other invariance properties to discrete structures. A discrete structure $\triangle$ is said to have a local inflation-deflation symmetry (LIDS) relative to a linear map $L$ if $\Delta$ and $L(\Delta)$ are MLD, $\Delta \stackrel{m l d}{\sim} L(\Delta)$. When $L(x)=\lambda x$ (or $L(x)=\lambda R x$ in general, with $R \in O(d, \mathbb{R})$ ), the number $\lambda$ is called the inflation multiplier of the LIDS. A necessary condition for L to define an LIDS is $Z(L(\Delta))=Z(\Delta)$. For tilings an inflation rule consists of the mappings

$$
\lambda T_{i} \mapsto \cup_{j=1}^{n} T_{j}+A_{i j}
$$

with finite sets $A_{i j} \subset \mathbb{R}^{d}$, subject to the mutual disjointness of the interiors of the sets on the right hand side and to the (individual) volume consistence condition $\operatorname{vol}\left(T_{i}\right)=\sum_{j=1}^{n} \operatorname{vol}\left(T_{j}\right) \operatorname{card}\left(A_{i j}\right)$, both for each $1 \leq i \leq n$. 


\section{Appendix B. 3-Dimensional Compound Quasicrystal from the $E_{8}$ Lattice}

The canonical coordinate system of the $E_{8}$-CQC can be give using an enumeration function. An enumeration function is an odd and growing function in and on $\mathbb{Z}$

$$
f_{V}: \mathbb{Z} \rightarrow \mathbb{Z} \mid\left(\forall x \in \mathbb{Z}, f_{V}(x)=-f_{V}(-x)\right) \text { and }\left(\forall x, y \in \mathbb{N}^{2}, f_{V}(x+y) \geq f_{V}(x)\right),
$$

and the canonical enumeration function is

$$
f_{V}(x)=\lfloor x \phi\rceil .
$$

The function $f_{V}(\mathbb{Z})$ is known as the OEIS sequence [48] and take the first 20 values: $\{0,2,3,5,6,8$, $10,11,13,15,16,18,19,21,23,24,26,28,29,31\}$. The density of $f_{V}(\mathbb{Z})$ in $\mathbb{Z}$, or the probability of an integer to be in the image of $f$ is $\delta(f)=\phi^{-1}$. Values for the function $f_{V}$ are given in Table (2). The interval function, or discrete derivative $d f_{V}(x)$ is the palindrome Fibonacci word encoded with Long=2 and Short=1, the sequence [49]

$$
d f_{V}(x)=f_{V}(x+1)-f_{V}(x)=\lfloor(x+1) \phi\rceil-\lfloor x \phi\rceil .
$$

The canonical coordinate system of the $E_{8}$-CQC is the image of $\mathbb{Z}^{3}$ by the canonical enumeration function: $f_{V}\left(\mathbb{Z}^{3}\right)$.

From the vertices found in the canonical coordinates, satisfying the Elser-Sloane conditions ( A20,A21,A22), the $E_{8}$-CQC selects only those forming regular tetrahedrons in the slice (corresponding to regular tetrahedrons in $E_{8}$.) One way to implement this is an elimination function, applied on $f_{V}\left(\mathbb{Z}^{3}\right)$, but it is proved that they don't use the full set $f_{V}(\mathbb{Z})$, so it is more efficient to compose the enumeration function $f_{V}$ with a second enumeration function $g_{T}$, where the composition gives $f_{T}$ At the next step we only keep in the $E_{8}$-CQC the "4-group", the tetrahedrons which meet as group of four at one common vertex such that their convex hull is a cuboctahedron. We know that they will correspond to the equator of a 24-cell in the $E_{8}$ Lattice. Again, one way to implement this is an elimination function, applied now on $f_{T}\left(\mathbb{Z}^{3}\right)$, but it is more efficient to compose the enumeration function with a new enumeration function $g_{4 G}$, to give $f_{4 G}$

$$
\begin{array}{r}
g_{4 G}(x)=((x \bmod 3)>0)\left\lfloor\phi^{(x \bmod 3)-3}(5(\lfloor\phi(\lfloor x / 3\rfloor+1)\rceil-\lfloor\phi\lfloor x / 3\rfloor\rceil)+3)\right\rceil \\
+5\lfloor\phi\lfloor x / 3\rfloor\rceil+3\lfloor x / 3\rfloor \\
f_{4 G}(x)=\left(f_{V} \circ g_{4 G}\right)(x)=f_{V}\left(g_{4 G}(x)\right) \\
f_{4 G}(x)=((x \bmod 3)>0)\left\lfloor\phi^{(x \bmod 3)-3}(8(\lfloor\phi(\lfloor x / 3\rfloor+1)\rceil-\lfloor\phi\lfloor x / 3\rfloor])+5)\right\rceil \\
+8\lfloor\phi\lfloor x / 3\rfloor\rceil+5\lfloor x / 3\rfloor
\end{array}
$$

Values for the function $f_{4 G}$ are given in Table (3).

Note that if we substitute 2 by $5,3,5$ and 1 by $3,2,3$ in the word (A13), we obtain $d g_{4 G}(\mathbb{Z})$ :

$$
d g_{4 G}(x)=g_{4 G}(x+1)-g_{4 G}(x)
$$

Further conditions are required for a point of the canonical coordinate system to belong to the $E_{8}-\mathrm{CQC}$, formulated in term of canonical elimination function. The elimination function has two steps. First, there is a planar elimination function selecting all acceptable pairs $\{x, y\}$ (where $\mathrm{x}$ and $\mathrm{y}$ are already images of an enumeration function, for example $f$ or $g$ ). This is a "sieve" process on $\mathbb{Z}^{2}$ or on any projection of a volumetric shape in which we want the model set texture. It has to be done once and is of order 2 while the whole volumetric computation is of order 3 of the size, and linear in the number of point obtained, that can be precisely predicted by the known density.

The step 1 mentioned above will give us a list of points $\{x, y\}$ in $f_{V}\left(\mathbb{Z}^{2}\right)$. The step 2 consists on computing a discrete fiber on each point obtained from step 1 . The fiber selection will be done through the volumetric elimination function depending on three variables, $f_{V}(x), f_{V}(y), f_{V}(z)$. 
Depending on the volumetric shape we are interested, $f_{V}(z)$ will be bounded between $\min f_{V}(z)$ and $\max _{V}(z)$ for each fiber. We define two functions of $x$ and $y$ valued in $\frac{\mathbb{Z}}{8 \mathbb{Z}}$ :

$$
\begin{aligned}
& h_{x 8}(x, y)=(-x+2 y-3\lfloor x / \phi\rceil-3\lfloor y / \phi\rceil) \bmod 8 \\
& h_{y 8}(x, y)=(-3 x-4 y-3\lfloor x / \phi\rceil+3\lfloor y / \phi\rceil) \bmod 8
\end{aligned}
$$

The values for $h_{x 8}(x, y)$ are explained in figures A1 and A2.

a.
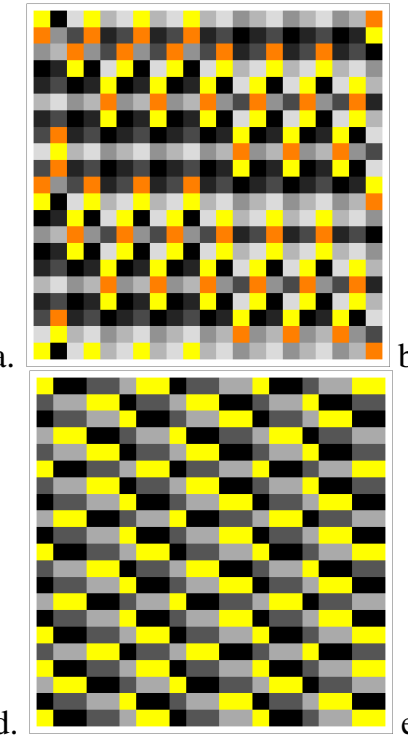

d.

Figure A1. a. $h_{x 8}(x, y), \quad$ b. $h_{y 8}(x, y)$,

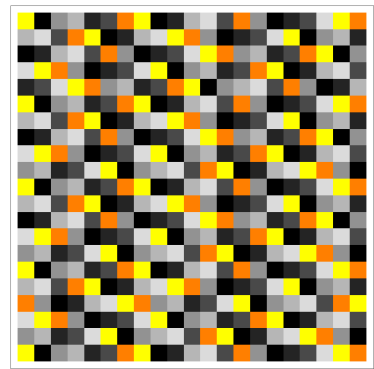

b.

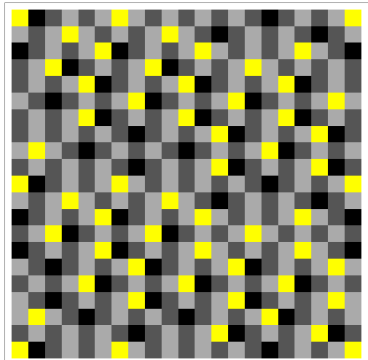

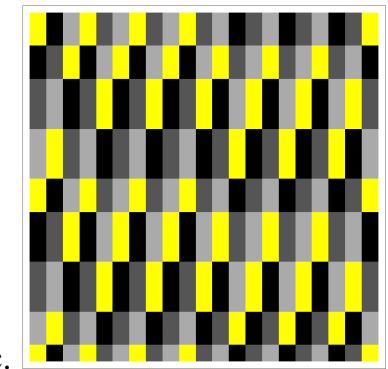

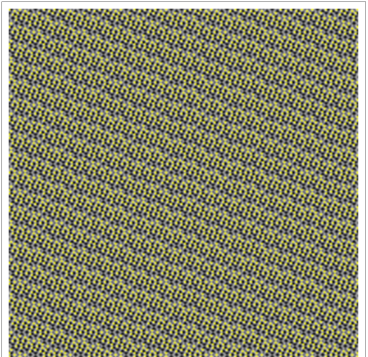
$h_{y 8}(x, y) \bmod 4$, f. like e for 200 points, the color yellow representing the value 0 and orange 4 of selected points

a.

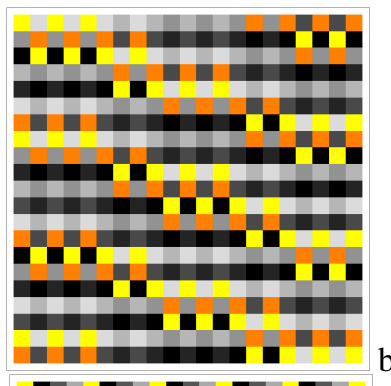

d.

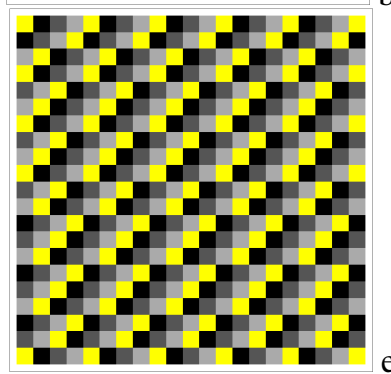

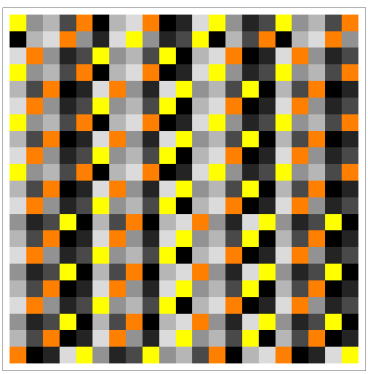
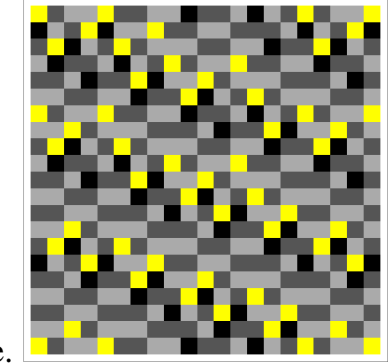

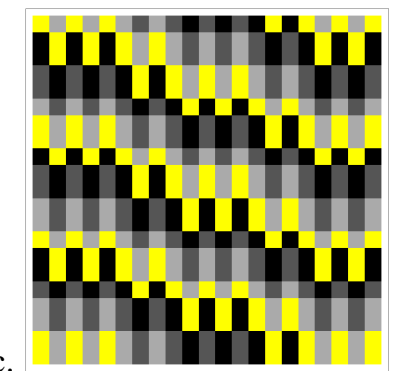

c.

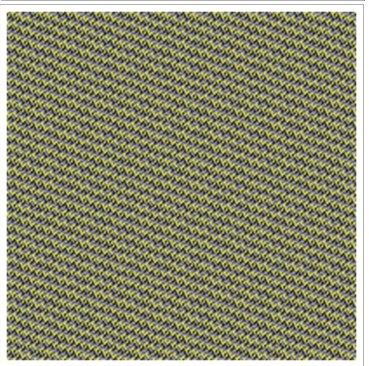

Figure A2. a. $h_{x 8} \circ f(x, y), \quad$ b. $h_{y 8} \circ f(x, y)$, c. $h_{x 8} \circ f(x, y) \bmod 4, \quad$ d. $h_{y 8} \circ f(x, y) \bmod 4$, e $h_{x 8} \circ f(x, y)+h_{y 8} \circ f(x, y) \bmod 4$, f. like e for 200 points, the color yellow representing the value 0 and orange 4 of selected points

$$
\mathbb{Z} "=\left\{x, y \in \mathbb{Z}^{\prime} \mid x m 8(x, y)=y m 8(x, y) \quad \& \quad y m 8(x, y) \equiv 0 \bmod 4\right\} .
$$

The density of $\mathbb{Z}$ ", the probability of a pair of integer to be in $\mathbb{Z}$ " is: $\delta_{\mathbb{Z}}=1 / 36$. The density of $\mathbb{Z}^{\prime}$ in $\mathbb{Z}^{\prime 2}$, the probability of an pair of $\mathbb{Z}^{\prime}$-integer to be in $\mathbb{Z}$ " is: $\delta_{\mathbb{Z}}^{\prime}$ " $=1 / 16$. 


$\begin{array}{cccccccccccc}x & 0 & 1 & 2 & 3 & 4 & 5 & 6 & 7 & 8 & 9 & 10 \\ x^{\prime} & 0 & 12 & 19 & 31 & 38 & 50 & 61 & 69 & 80 & 92 & 99 \\ x^{\prime} & 0 & 6 \phi+2 & 8 \phi+6 & 14 \phi+8 & 16 \phi+12 & 22 \phi+14 & 28 \phi+16 & 30 \phi+20 & 36 \phi+22 & 42 \phi+24 & 44 \phi+28 \\ x^{\prime} & 0 & 11.708 & 18.944 & 30.652 & 37.889 & 49.597 & 61.305 & 68.541 & 80.249 & 91.957 & 99.193\end{array}$

Table 1: Table of values for $x, x^{\prime}, x^{\prime} \in \mathbb{D}, x^{\prime} \in \mathbb{R}$

We may use the shortcut notation $\mathbb{Z}^{\prime}=\mathbb{Z} \pi^{1}$ and $\mathbb{Z} "=\mathbb{Z} \pi^{2}$ to exhibit the analogy between subsets and self-projective lines or planes.

1. The canonical planar elimination function $h(x, y)$ is encoding the fact that the $E_{8}-\mathrm{CQC}$ is a slice of an Elser-Sloane projection of the $E_{8}$ Lattice:

$$
h(x, y)=h_{x 8}(x, y) \equiv 0(\bmod 4) \wedge h_{y 8}(x, y) \equiv 0(\bmod 4)
$$

2. The canonical volumetric elimination function $h(x, y, z)$ is encoding the fact that the $E_{8}$-CQC is a slice of an Elser-Sloane projection of the $E_{8}$ Lattice:

$$
h(x, y, z)=\left(\begin{array}{cccccc}
-1 & 2 & 0 & -3 & -3 & 0 \\
-1 & -4 & 2 & 1 & 1 & 2 \\
-3 & -4 & 2 & -3 & 1 & -4 \\
-1 & -4 & -4 & 1 & -1 & -2 \\
-1 & 2 & 2 & -1 & -3 & 2 \\
1 & 2 & 2 & 3 & -3 & 0 \\
-3 & -4 & 0 & -3 & 3 & 0 \\
-3 & 2 & -4 & -3 & -3 & -2
\end{array}\right) .\{x, y, z,\lfloor x / \phi\rceil,\lfloor y / \phi\rceil,\lfloor z / \phi\rceil\} \equiv(0 \| 4)(\bmod 8)
$$

(so either the 8 values of the vector are congruent to 0 modulo 8 , or they are all congruent to 4 modulo 8, the matrix given in equation (A21) is deduced from the orientation of the six dimensional subspace of $E_{8}$ projecting by Elser-Sloane projection to the selected 3-dimensional slice in which $x^{\prime}, y^{\prime}, z^{\prime}$ is living)

3. The canonical spherical elimination function $s(x, y, z)$ is encoding the fact that the star map image is restricted to a sphere of radius $\phi-1$

$$
s(x, y, z)=\star(x)^{2}+\star(y)^{2} \star(w)^{2}<r^{2}
$$

Finally the model set coordinates $x^{\prime}, y^{\prime}, z^{\prime}$ in $\mathbb{D}^{3}$, and the coordinates $x ", y$ ", $z$ " in $\mathbb{Z}^{3}$, here called crystal proxy, are deduced from the set of $\{x, y, z\}$ by the bijections between $x, x^{\prime}$ and $x^{\prime \prime}$ (and the same for $y$ and $z$ ).

$$
\begin{aligned}
x^{\prime}(x) & =10 x-\sqrt{20} \star(x) \\
x\left(x^{\prime}\right) & =\left\lfloor x^{\prime} / 10\right\rceil \\
x^{\prime}(x) & =10 x-\lfloor\sqrt{20} \star(x)\rceil \\
x\left(x^{\prime \prime}\right) & =\left\lfloor x^{\prime \prime} / 10\right\rceil \\
x^{\prime}\left(x^{\prime \prime}\right) & =10\left\lfloor x^{\prime \prime} / 10\right\rceil-\sqrt{20} \star(\lfloor x " / 10\rceil) \\
x^{\prime}\left(x^{\prime}\right) & =\left\lfloor x^{\prime}\right\rceil
\end{aligned}
$$

Numerical values are given in Table(1).

The last equation defines and justifies the name of crystal proxy for the object in the doubleprime coordinate. It is embedded in $\mathbb{Z}^{3}$ (or $\mathbb{Z}^{n}$ for a generalization), and therefore a subset of a lattice or crystal, but it is at a distance smaller than one in each coordinate from the exact model set point in prime coordinates. Crystal proxy coordinates are advantageously and efficiently used for graphics representations of the model sets, because the differences are close to invisible.

We refer you to [50] for deeper insight in python and Wolfram language implementations. 


$\begin{array}{ccccccccccc}k & 0 & 1 & 2 & 3 & 4 & 5 & 6 & 7 & 8 & 9 \\ x=f_{V}(k) & 0 & 2 & 3 & 5 & 6 & 8 & 10 & 11 & 13 & 15 \\ x^{\prime \prime} & 0 & 19 & 31 & 50 & 61 & 80 & 99 & 111 & 130 & 149 \\ x^{\prime} & 0 & 8 \phi+6 & 14 \phi+8 & 22 \phi+14 & 28 \phi+16 & 36 \phi+22 & 44 \phi+28 & 50 \phi+30 & 58 \phi+36 & 66 \phi+42 \\ x^{\prime} & 0 & 18.944 & 30.652 & 49.597 & 61.305 & 80.249 & 99.193 & 110.90 & 129.85 & 148.79 \\ \star(x) & 0 & 2 \phi-3 & 3 \phi-5 & 5 \phi-8 & 6 \phi-10 & 8 \phi-13 & 10 \phi-16 & 11 \phi-18 & 13 \phi-21 & 15 \phi-24 \\ \star(x) & 0 & 0.23607 & -0.14590 & 0.090170 & -0.29180 & -0.055728 & 0.18034 & -0.20163 & 0.034442 & 0.27051\end{array}$

Table 2: Table of values for $x, x^{\prime \prime}, x^{\prime}, \star(x)$ for $x=f_{V}(k)$ such that $\star(x)<\frac{1}{2} \phi^{-1}$

$\begin{array}{cccccccccc}k & 0 & 1 & 2 & 3 & 4 & 5 & 6 & 7 & 8 \\ x=f_{4 G}(k) & 0 & 8 & 13 & 21 & 26 & 29 & 34 & 42 & 47 \\ x^{\prime \prime} & 0 & 80 & 130 & 210 & 260 & 290 & 340 & 420 & 470 \\ x^{\prime} & 0 & 36 \phi+22 & 58 \phi+36 & 94 \phi+58 & 116 \phi+72 & 130 \phi+80 & 152 \phi+94 & 188 \phi+116 & 210 \phi+130 \\ x & 0 & 80.249 & 129.85 & 210.10 & 259.69 & 290.34 & 339.94 & 420.19 & 469.79 \\ x * & 0 & 8 \phi-13 & 13 \phi-21 & 21 \phi-34 & 26 \phi-42 & 29 \phi-47 & 34 \phi-55 & 42 \phi-68 & 47 \phi-76 \\ x * & 0 & -0.0557 & 0.0344 & -0.0212 & 0.0688 & -0.0770 & 0.0131 & -0.0425 & 0.0475\end{array}$

Table 3: Table of values for $x, x^{\prime \prime}, x^{\prime}, x *=\star(x)$ for $x=f_{4 G}(k)$ such that $\star(x)<\frac{3}{2} \phi^{-7}$

\section{Appendix C. Aperiodic Functions}

As discussed in the main text, one implication of having an underlying structure such as the proposed model sets is that one has to describe general relational evolution with regard to such clock structures. The more obvious properties of models sets are in most cases described by aperiodic functions, which goes back to [51]. An general example of this kind of function is given by $\sin (2 \pi x)+\cos (2 \pi \alpha x)$ with $\alpha$ an irrational number. This aperiodic function is related to a $Z^{2}$-periodic function of two variables via $\sin (2 \pi x)+\left.\cos (2 \pi y)\right|_{y=\alpha x}$. Using such a function would amount to have one aperiodic clock in lower dimension or to deal with a notion of higher dimensional clocks. Let us see some concrete examples from model sets. For a sample of a point set of $E_{8}$-CQC derived by using 6-dimensional canonical windows we get a relation between distance in parallel space and perpendicular space as shown in Figure A3.

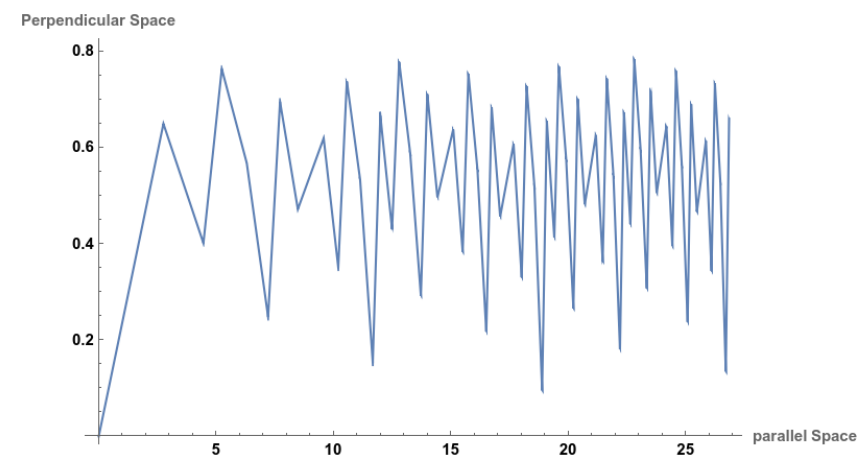

Figure A3. Distance in parallel space versus perpendicular space for $E_{8}$-CQC.

Consider as a second example the two point correlation function for hits, Eq. (21), which depends on products of $H_{i j}^{K_{i}}$ weights Eq. (13), $E^{h}\left[H_{i j}^{K_{i}}\right]$, where the spherical approximation of the windows allows us to compute it as function of the distance between $\kappa_{i}$ and $\kappa_{j}$ for the same window of fixed radius, such as in Eq. (30). This is equivalent to fix one inflation window spherical approximation with center at origin and radius $R_{0}, K_{R_{0}}^{i}$, and translating a copy to distance $d_{0 j}$, with $H_{0 j}^{h}(d)=\left(\operatorname{Vol}\left(K_{R_{0}}^{i}\right) \cap\left(d^{\star}+K_{R_{0}}^{i}\right)\right) / \operatorname{Vol}\left(K_{R_{0}}^{i}\right)$ and with $d_{0 j}$ such that $d^{\star} \subset K_{R_{0}^{l}}$ and $K_{R_{0}^{l}}$ the spherical approximation of the larger cut window $K_{K}^{l}$, given $E^{h}\left[H_{i j}^{K_{i}}\right] \sim \prod_{l} H_{0 l}^{h}$. In terms of indicator functions $1_{K}$ we have $H_{0 j}^{h}(d)=\frac{1}{\operatorname{Vol}\left(K_{R_{0}}^{i}\right)} \int_{K_{R_{0}}^{i}} 1_{K_{R_{0}}^{i}}(y) 1_{\left(d^{\star}+K_{R_{0}}^{i}\right)}(y) d y$, which has well 
known Fourier transform, $\widehat{H_{0 j}^{h}}(\bar{d})=|\mathcal{A}(\bar{d})|^{2}$, with $\bar{d} \in \bar{L}$, the projection to parallel space of the dual embedding lattice, and with the amplitude $\mathcal{A}(\bar{d})$ given by

$$
\mathcal{A}(\bar{d})=\frac{1}{\operatorname{Vol}\left(K_{R_{0}}^{i}\right)} \int_{K_{R_{0}^{i}}} e^{2 \pi i y \bar{d}^{\star}} d y=\frac{3(\sin (z)-z \cos (z))}{(z)},
$$

where $z=2 \pi R_{0}\left|\bar{d}^{\star}\right|$. Figure A4 gives the general form of the Fourier transform of $E^{h}\left[H_{i j}^{K_{i}}\right]$, which depends on $R_{0}$ of specific $\kappa_{i}$ and the average distance between $\kappa_{i}$ 's in a specific model set.

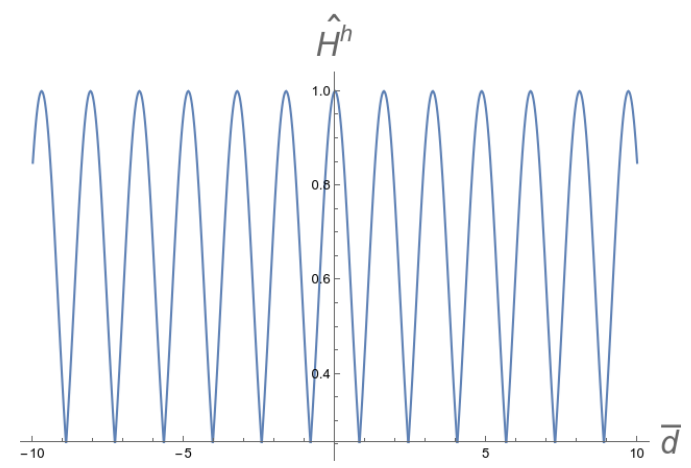

Figure A4. The Fourier transform of expected value $E^{h}\left[H_{i j}^{K_{i}}\right]$.

Another example, which could give an aperiodic discrete clock, is given by the diffraction spectrum, Eq. (A5), where the intensities coefficients are given from Eq. (A29). The diffraction for the $E_{8}-\mathrm{CQC}$ is shown in Figure A5

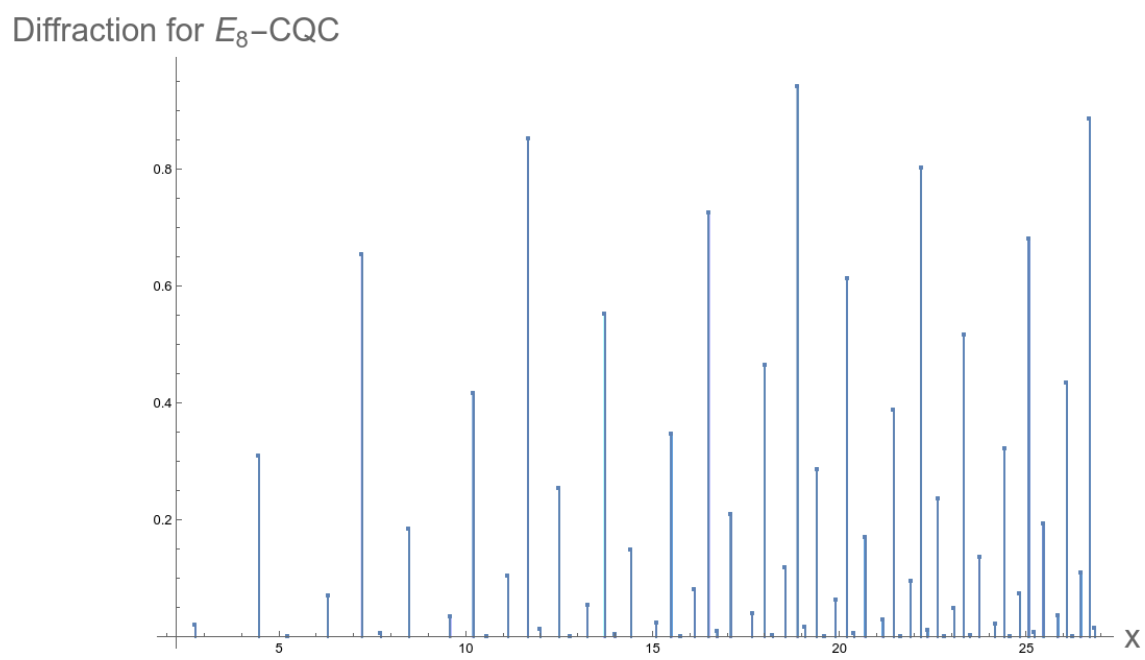

Figure A5. The diffraction for $E_{8}-\mathrm{CQC}$ as function of the norm in parallel Space $(x)$.

\section{References}

1. Verlinde, E. On the origin of gravity and the laws of Newton. J. High Energ. Phys. 2011, 29 (2011).

2. Dieks, D., van Dongen, J., de Haro, S. Emergence in Holographic Scenarios for Gravity, Stud. Hist. Phil. Sci. B 52, 203-216 (2015).

3. Donà, P., Fanizza, M., Sarno, G., Speziale, S. SU(2) graph invariants, Regge actions and polytopes, Class. Quant. Grav. 35, no.4, 045011 (2018) doi:10.1088/1361-6382/aaa53a [arXiv:1708.01727 [gr-qc]].

4. Steinhaus, S. Coarse Graining Spin Foam Quantum Gravity_A Review, Front. in Phys. 8, 295 (2020). [arXiv:2007.01315 [gr-qc]].

5. Gozzini, F. A high-performance code for EPRL spin foam amplitudes,” Classical and Quantum Gravity, (2021). [arXiv:2107.13952 [gr-qc]].

6. Ambjorn, J., Jurkiewicz, J., Loll, R. Causal Dynamical Triangulations and the Quest for Quantum Gravity, (2010). [arXiv:1004.0352 [hep-th]].

7. Cooperman, J. H., Making the case for causal dynamical triangulations, Found Phys 50, 1739-1755 (2020). [arXiv:1410.0670 [gr-qc]].

8. Bombelli, L., Lee, J., Meyer, D., Sorkin, R. D. Space-time as a causal set. Physical review letters, 59(5):521, (1987).

9. Bolognesi, T. Spacetime computing: towards algorithmic causal sets with special-relativistic properties. In Advances in Unconventional Computing, pages 267-304. Springer, 2017. 
10. Wolfram, S. A class of models with the potential to represent fundamental physics, arXiv:2004.08210 [cs.DM], (2020).

11. Gorard, J. Some Relativistic and Gravitational Properties of the Wolfram Model, arXiv:2004.14810v1 [cs.DM], (2020).

12. Arsiwalla, X. D., Gorard, J. Pregeometric Spaces from Wolfram Model Rewriting Systems as Homotopy Types, arXiv:2111.03460 [math.CT], (2021).

13. Leuenberger, G. Emergence of Minkowski-Spacetime by Simple Deterministic Graph Rewriting, arXiv:2110.03388 [gr-qc], (2021).

14. 't Hooft, G. Fast Vacuum Fluctuations and the Emergence of Quantum Mechanics. Found Phys 51, 63 (2021).

15. Baake, M., Grimm, U. Aperiodic Order, Cambridge University Press, (2013).

16. Moody, R. V. Model sets: A Survey, [arXiv:math/0002020 [math.MG]].

17. Senechal, M. J., Quasicrystals and Geometry, Cambridge University Press, (1995).

18. Levine, D., Steinhardt, P. J., Quasicrystals. I. Definition and structure. Phys Review B, 1986, 34, 596.

19. Takakura, H., Gomez, C., Yamamoto, A. et al. Atomic structure of the binary icosahedral Yb-Cd quasicrystal. Nature Mater 6, 58-63 (2007).

20. Amaral, M.; Fang, F.; Hammock, D.; Irwin, K. Geometric State Sum Models from Quasicrystals. Foundations 2021.

21. Amaral, M. M., Bojowald, M. A path-integral approach to the problem of time, Annals Phys. 388, 241-266 (2018). [arXiv:1601.07477 [gr-qc]].

22. Bojowald, M., Martinez, L., Wendel, G., Relational evolution with oscillating clocks, arXiv:2110.07702 [quant-ph].

23. Irwin, K. Toward the Unification of Physics and Number Theory. Reports in Advances of Physical Sciences, 3, $1950003,2019$.

24. Langan, C. M. The Cognitive-Theoretic Model of the Universe: A New Kind of Reality Theory. Progress in Complexity, Information and Design, 2002.

25. Irwin, K. The Code-Theoretic Axiom: The Third Ontology. Reports in Advances of Physical Sciences, 3, 1950002, 2019.

26. Irwin, K.; Amaral, M.; Chester, D. The Self-Simulation Hypothesis Interpretation of Quantum Mechanics. Entropy 2020, $22,247$.

27. Hammock, D.; Fang, F.; Irwin, K. Quasicrystal Tilings in Three Dimensions and Their Empires. Crystals, $2018,8,370$.

28. Fang, F., Irwin, K. An Icosahedral Quasicrystal and E8 derived quasicrystals. arXiv:1511.07786v2 [math.MG]. (2016).

29. Elser, V., Sloane, N. J. A. A highly symmetric four-dimensional quasicrystal, J. Phys. A, 20:6161-6168, (1987).

30. Chen, L., Moody, R. V., Patera, J. Non-crystallographic root systems, In: Quasicrystals and discrete geometry. Fields Inst. Monogr, (10), (1995).

31. Amaral, M. M., Aschheim, R., Bubuianu, L., Irwin, K., Vacaru, S. I, Woolridge, D., Anamorphic Quasiperiodic Universes in Modified and Einstein Gravity with Loop Quantum Gravity Corrections, Class. Quant. Grav. 34, no.18, 185002 (2017). [arXiv:1611.05295 [gr-qc]].

32. Kuchař, K. V., Time and interpretations of quantum gravity, In G. Kunstatter, D. E. Vincent, and J. G. Williams, editors, Proceedings of the 4th Canadian Conference on General Relativity and Relativistic Astrophysics, Singapore, 1992. World Scientific.

33. Isham, C. J., Canonical Quantum Gravity and the Question of Time, In J. Ehlers and H. Friedrich, editors, Canonical Gravity: From Classical to Quantum, pages 150-169. Springer-Verlag, Berlin, Heidelberg, 1994.

34. Anderson, E., The Problem of Time in Quantum Gravity, In V. R. Frignanni, editor, Classical and Quantum Gravity: Theory, Analysis and Applications. Nova, New York, 2012, [arXiv:1009.2157].

35. Bojowald, M., Höhn, P. A., Tsobanjan, A. An effective approach to the problem of time: general features and examples, Phys. Rev. D 83, 125023, (2011), [arXiv:1011.3040].

36. Dirac, P., A., M., Generalized Hamiltonian dynamics, Can. J. Math. 2 129-148, (1950).

37. Rovelli, C., Why Gauge?, Foundations of Physics, 44 (2014) no. 1, 91-104, arXiv:1308.5599.

38. Amaral, M., M., Some remarks on relational nature of gauge symmetry, urlhttp://philsci-archive.pitt.edu/10995, (2014).

39. Wendel, G., Martinez, L., Bojowald, M. Physical implications of a fundamental period of time, Phys. Rev. Lett. 124, no.24, 241301 (2020). [arXiv:2005.11572 [gr-qc]].

40. Bellissard, J. Gap labelling theorems for Schrödinger's operators. In: From Number Theory to Physics, pp. 538-630, Les Houches March 89, Springer, J.M. Luck, P. Moussa \& M. Waldschmidt Eds., (1992).

41. Kleinert, H. Path integrals in quantum mechanics, statistics, polymer physics, and financial market. 5th edition, World Scientific, (2009).

42. Fang, F.; Clawson, R.; Irwin, K. The Curled Up Dimension in Quasicrystals. Crystals, 11, 1238, (2021).

43. Tegen, R., Relativistic Harmonic Oscillator Potential for Quarks in the Nucleon, Annals Phys. 197, 439-455 (1990).

44. Alexander, S., Cunningham, W. J., Lanier, J., Smolin, L., Stanojevic, S., Toomey, M. W., Wecker, D. The Autodidactic Universe, [arXiv:2104.03902 [hep-th]].

45. Vanchurin, V., Towards a theory of quantum gravity from neural networks. arXiv:2111.00903 [cs.LG], (2021).

46. 't Hooft, G. The Black Hole Firewall Transformation and Realism in Quantum Mechanics. Universe. 2021; 7(8):298. arXiv:2106.11152v1 [gr-qc], (2021).

47. Billingsley, P. Probability and measure / Patrick Billingsley Anniversary ed. Wiley series in probability and mathematical statistics. John Wiley \& Sons (2012).

48. https://oeis.org/A007067

49. Hofstadter, D. https://oeis.org/A006340, (1977).

50. Aschheim, R, et al. Aperiodic textures, work in progress, (2021).

51. Bohr, H., Almost Periodic Functions, reprint (Chelsea, New York), (1947). 